\title{
Robust Planning and Operation of Multi-Cell Homogeneous and Heterogeneous Networks
}

\author{
Yiğit Özcan ${ }^{\dagger}$, Jad Oueis*, Catherine Rosenberg ${ }^{\dagger}$, Razvan Stanica*, Fabrice Valois* \\ ${ }^{\dagger}$ Department of Electrical and Computer Engineering, University of Waterloo, Canada \\ *Univ Lyon, INSA Lyon, Inria, CITI, F-69621 Villeurbanne, France
}

\begin{abstract}
In this work, we propose a robust planning tool that allocates power statically in homogeneous and heterogeneous cellular networks with non-regular base station (BTS) placement, to mitigate interference and improve overall performance. Each BTS will use the total available spectrum, but it will divide it into multiple sub-bands, and each BTS will transmit with a specific pre-computed power on each sub-band. We refer to such a power allocation as a power map. Our offline planning tool computes a robust power map for a given topology, by solving a non-convex, non-linear optimization problem, through simple transformations, based on geometric programming. The power map is computed based solely on the network topology, and it is made available to all BTSs that use it throughout the network operation to perform scheduling using a fast quasi-optimal online algorithm that we propose. We evaluate our planning tool for different homogeneous and heterogeneous networks (HetNets), first in a static setting where scheduling is performed optimally and then in a dynamic setting when scheduling is performed with our online scheduler. Results show that our solution significantly outperforms a classical equal power/fixed frequency reuse scheme in terms of sum-rate, by up to $30 \%$ in homogeneous networks and by up to $70 \%$ in HetNets.
\end{abstract}

Index Terms-Power Map, Geometric Programming, Cellular Networks, Heterogeneous Networks, Scheduling

\section{INTRODUCTION}

One of the main objectives of $5 \mathrm{G}$ is to alleviate the spectrum scarcity problem suffered by cellular networks. However, we believe that spectrum will always remain a scarce resource that needs to be managed well. Hence, there is a need to develop a complete and efficient suite of radio resource management (RRM) processes that are easy to implement. This suite includes channel allocation, power allocation, scheduling, user allocation, and interference mitigation techniques.

In this work, we focus on power and channel allocation on the downlink of a multi-cell orthogonal frequency division multiple access (OFDMA) wireless network. These processes are usually performed in a planning or upgrade phase offline, although power can be also allocated in a more dynamic way (e.g., through scheduling), but at a very high complexity cost. A simple state of the art (SoA) planning benchmark is one where every base station (BTS) uses the complete frequency band and spreads its power budget equally on every subchannel. This might not be an efficient solution, especially in non-regular networks where interference might become prohibitive for some users.

Most studies on cellular networks are based on the common optimal planar mobile network, modeled with BTSs placed in a uniform-size hexagonal grid [1], [2]. One of the particularities of our work is that we study networks with non-regular BTS placement, instead of assuming a deterministic BTS placement on a regular grid. In practice, network deployments in urban and suburban areas are seemingly more random [3] than ideally organized, and cell coverage radii are highly variable, depending on transmission power differences, tower heights, and user density [4]. They are also heterogeneous, i.e., with low and high power BTSs, creating even more complex situations. In such networks, the number of interfering BTSs and the amount of interference are highly variable from one network area to the other [5]. The coverage problem in regular BTS deployments is simple (e.g., using the SoA planning benchmark described above) and hence, most of the papers focus only on performance. In non-regular networks, the problem of coverage is difficult and cannot be under-estimated. Hence, planning non-regular networks is intuitively not a simple task and operating such a network using the SoA planning benchmark described above is not a good idea.

In this paper, we consider a system with non-regular (possibly heterogeneous) BTS placement in which all BTSs use the complete spectrum, and we tackle the problem of robust power allocation. To this end, we assume that the frequency band is divided into sub-bands, and each BTS will transmit with a specific pre-computed power on each sub-band. The first challenge, in this case, is to parametrize the power map offline, i.e., to find a suitable number of sub-bands, and to assign the corresponding power levels on each sub-band, keeping in mind the non-regular cell pattern and the possible heterogeneity of the network. The selection of these parameters should be done with robustness in mind, i.e., it should allow good performance for a variety of user distributions and/or densities. This is a desirable feature since changing the power allocation very frequently depending on the network state is not very realistic. The second challenge is to design a scheduling algorithm that takes the power map into account and is efficient, fair and easy to implement in real-time.

The contributions of this paper are as follows. First, we propose an offline planning tool, both for homogeneous and heterogeneous networks with non-regular BTS placement, that outputs a robust power map to be followed by the BTSs, for a given network topology and a given number of sub-bands. In other words, the tool determines with which power should each BTS transmit on each sub-band. The power map is computed prior to the actual network deployment, based solely on the network topology, with no accurate information on the 
user locations. Then, it is fixed and followed by the BTSs throughout the network operation. We show that this power map is robust, in the sense that it performs well independently of the actual network state, such as the number of users in the system, their distribution, and their evolution. The proposed tool solves a non-convex, non-linear, complex optimization problem, through multiple but simple transformations, based on signomial and geometric programming.

Second, since the proposed algorithm that outputs the power map depends on several input parameters, we assess its performance with respect to those parameters in homogeneous systems and, then, in heterogeneous systems. Specifically, we study the impact on the power map performance of each of these parameters and we give some insights on how to set them, notably the number of sub-bands. We also quantify the gain in performance that our robust power map scheme brings with respect to SoA benchmarks. This study is done on given snapshots of the network with different numbers of users already associated to BTSs, and for different network sizes and topologies.

Note that planning and managing a HetNet is even more challenging than planning and managing a homogeneous network, since it requires careful user association and channel allocation schemes due to the large power budget difference between the macro (high power) BTSs and the small cells (the low power BTSs). We show that our robust power map scheme works very well in a HetNet context and outperforms the benchmark schemes we study, significantly.

Finally, we evaluate our method on dynamic scenarios, by simulating users arriving, associating with a BTS, downloading a file and departing, over a period of time. To this end, we propose an online local scheduling algorithm that does not require complex computations. We show that this scheduling algorithm performs quasi-optimally. Our final results show that our power map scheme, parametrized solely knowing the network topology, significantly outperforms the benchmarks in terms of average delay for both homogeneous and heterogeneous networks.

In Sec. II, we discuss related work. In Sec. III, we describe the network model and the notion of a power map. We present the algorithm to parametrize a power map in Sec. IV. In Sec. V, we present numerical results for static settings, first for homogeneous networks, by studying the impact of each input parameter, and then for HetNets. In Sec. VI, we move to dynamic settings, propose an online scheduling algorithm that uses the power map and evaluate the resulting performance both for homogeneous and heterogeneous networks. We conclude the paper in Sec. VII.

\section{RELATED WORK}

Inter-cell interference (ICI) is a major problem in OFDMA-based systems, occurring when neighboring BTSs are allocated the same frequency bands [6]. A multitude of inter-cell interference coordination (ICIC) techniques have been developed, aiming to reduce ICI [7]. Note that considering a single cell network in the power allocation problem, such as in [8], does not take into account ICI and hence is not practical.
One way to achieve ICIC is through centralized coordinated scheduling. However, a fully centralized scheduler is complex to implement in practice [9], since it requires all the channel gain information in the system and it cannot be computed fast enough in a real time system, where scheduling is performed every few milli-seconds. Several distributed schedulers have been proposed [10], [11]. Such schemes require a large amount of information exchange between BTSs very frequently, which is not really feasible either.

A number of studies have focused on downlink power allocation assuming regular BTS deployment. Authors of [12] focus on energy efficiency, where the BTSs coordinate with each other. The proposed scheme requires frequent data exchange and also frequent power adjustment. Sum-rate maximization is studied in [13], which lacks fairness among the users. A computationally expensive optimization algorithm and a sub-optimal heuristic solution are proposed in [14] for fractional frequency reuse. The proposed scheme is based on sum-rate maximization and does not consider robustness as it needs to be updated for every realization. A dynamic power allocation for sum-rate maximization is studied for OFDMA networks in [15], which is also per-realization based. An iterative algorithm is proposed in [16] for power minimization, however fairness among users is not considered. Note that non-regular BTS placement introduces additional challenges such as non-regular cell serving area (and hence a coverage problem), as well as different interference patterns due to different number of interfering BTSs for each cell.

Authors of [17]-[19] study downlink performance using stochastic geometry, focusing on the average behavior over all possible realizations of a non-regular network. However, these studies take a macroscopic view and do not focus in detail on low-level processes such as scheduling.

Various frequency reuse schemes exist, more practical and easier to implement than optimized schedulers [6]. One of the ICIC techniques proposed to improve spectral efficiency is fractional frequency reuse (FFR) [20]. There are two common FFR deployment modes: strict FFR and soft frequency reuse (SFR). In strict FFR [21], a set of channels are re-used by all BTSs and allocated to users in the cell-center area only, while the remaining channels, allocated for users in cell-edge areas, are partitioned across BTSs based on a reuse factor $r$, similarly to a fixed frequency reuse scheme. This lowers ICI while slightly improving spectrum efficiency with respect to a strict reuse [22] but the spectrum can still be under-utilized [6].

SFR [1], [23] introduces the tuning of BTS transmission power levels as a mean to reduce ICI. Each BTS can utilize the entire bandwidth, eliminating the loss in spectral efficiency that is inherent in strict FFR, while improving the system performance [24]. The advantage of SFR has been demonstrated, mostly in hexagonal-grid homogeneous networks [1], [24], [25], [39], but no effort has been made to generalize SFR to HetNets and to produce an efficient and fast online scheduler to work with it. Moreover, SFR only considers three bands and two power levels, i.e., a high and a low power level at each sub-band, and hence has a very limited flexibility. What we propose in this paper can be considered as a generalization of the traditional SFR method, as we do 
not put any constraint on the number of sub-bands and the power levels, and we propose a method to parametrize it. In the following, we will consider the traditional SFR as one of our benchmarks and compare the performance of the proposed method with SFR in Sec. V-A3. We will also explain why SFR cannot be used as is in a HetNet, while our method is versatile enough to be used.

There has been much work on HetNets focusing on different RRM processes. As will be explained later in this paper, processes that are rather simple in homogeneous networks, such as user association and channel allocation, are more complex in HetNets, due to the imbalance between the power budgets of the BTSs [26]. Power minimization is studied in a HetNet with one macro BTS and one small cell in [27]. Sum-rate maximization is studied for HetNets in [13]. An iterative algorithm is proposed in [28] for power allocation and user association in HetNets. Most of the work on power management in HetNets [30]-[33] relies on a huge information exchange among the BTSs, which is not actually feasible in a real network. Also, the solutions proposed in the SoA are not robust, i.e., they do not work well for different realizations.

Simple channel allocation methods for HetNets have been studied in [34]. It has been shown that, when all the small cells and the macro BTSs operate on the whole frequency band and the power budget of a BTS is shared equally among all subchannels, the throughput of the users connected to the small cells is very low since they perceive very high interference from the macro BTSs. It was also shown that, when the small cells and the macro BTSs operate on separate bands, the performance can be significantly increased. In the following, we will study HetNets and show that the performance can be drastically improved when all the BTSs can operate on the same band, if we can compute a robust power map beforehand. Note that our study is novel in the sense that we focus on robust power allocation together with user scheduling and user association, both in static and dynamic settings in a HetNet with non-regular BTS deployment, while taking fairness into account. Note that even if we assume that the BTSs can exchange all the channel information in real time, i.e., the channel gain between each user-BTS pair, a network-level efficient power allocation requires complex computations, which cannot be done in the BTSs (even in powerful servers) in real time. We will show that using a pre-computed power map allows for the real time operation of a network in a very efficient way.

\section{SYSTEM MODEL}

In this section, we describe the static system model, on which the first part of this work is based (the second part of the work uses a dynamic framework). The notations used throughout the paper are summarized in Table I.

We consider a snapshot of a cellular network, where $\mathcal{J}$ is the set of BTSs and $\mathcal{U}$ is the set of deployed users. Note that all the BTSs in $\mathcal{J}$ are macro BTSs for the homogeneous case, whereas a BTS in $\mathcal{J}$ can be either a small cell or a macro BTS for the HetNet case. Given a user association strategy, let $\mathcal{U}_{j}$ be the set of users in cell $j$. We consider an OFDM-based
TABLE I

TABLE OF NOTATIONS

\begin{tabular}{|c|c|}
\hline $\mathcal{J}$ & Set of BTSs \\
\hline $\mathcal{U}$ & Set of users \\
\hline $\mathcal{U}_{j}$ & Set of users associated to BTS $j$ \\
\hline$M$ & Total number of channels \\
\hline$T$ & Number of subframes in a frame \\
\hline$P_{j}$ & Power budget of BTS $j$ \\
\hline $\mathcal{S}$ & Set of sub-bands \\
\hline$b$ & Total number of sub-bands \\
\hline$k$ & Number of channels per sub-band \\
\hline$P_{j}^{s}$ & Transmission power of BTS $j$ on the channels of a sub-band $s$ \\
\hline$r$ & Frequency reuse factor \\
\hline$\gamma_{u, j}^{s}$ & SINR per channel between user $u$ and BTS $j$ on sub-band $s$ \\
\hline $\mathcal{N}_{0}$ & Noise power per subchannel \\
\hline$G_{u, j}$ & Channel gain between a user $u$ and BTS $j$ \\
\hline$D_{u, j}$ & Distance between a user $u$ and BTS $j$ \\
\hline$\Gamma_{u, j}$ & Path loss between a user $u$ and BTS $j$ \\
\hline$G^{a}$ & Antenna gain \\
\hline$L$ & Equipment losses \\
\hline$R_{u, j}^{s}$ & Rate seen by user $u$ of BTS $j$ on sub-band $s$ \\
\hline$\alpha_{u, j}^{s}$ & Proportion of time sub-band $s$ is allocated to user $u$ on BTS $j$ \\
\hline$\lambda_{u}$ & Throughput of user $u$ \\
\hline$\Lambda$ & Throughput geometric mean in a network \\
\hline$\Omega$ & Set of calibration realizations $\omega$ \\
\hline$N_{\omega}$ & Number of users in a calibration realization $\omega$ \\
\hline$\Pi$ & Set of test snapshots $\pi$ \\
\hline$N_{\Pi}$ & Number of users in test snapshots $\pi$ \\
\hline$\epsilon$ & Small positive number \\
\hline$\phi$ & User association parameter for HetNets \\
\hline
\end{tabular}

system with a total of $M$ orthogonal channels, allocated to the set of BTSs and a time frame made of $T$ subframes. We use the terms channels and subchannels interchangeably in the paper. Channels are assumed to be flat within a frame, i.e., the channel gains across different channels between a pair of nodes are equal. We denote the power budget of BTS $j$ as $P_{j}$.

We assume that the frequency band is partitioned into non-overlapping sets of sub-bands, of equal size $k$. There are $b$ sub-bands (i.e., $k=M / b$ ). Let $\mathcal{S}$ be the set of those $b$ sub-bands where band $s_{n}(n \in\{1, \ldots, b\})$ contains channels $(n-1) k+1$ to $n k$. All BTSs can transmit on all sub-bands, with a different transmission power on each sub-band. We denote by $P_{j}^{s}$ the power budget of BTS $j \in \mathcal{J}$ on sub-band $s \in \mathcal{S} . P_{j}^{s} / k$ is the per channel power used on all the $k$ subchannels of sub-band $s$. A power map is characterized by $b$, the set $\mathcal{S}$, and the set of all $P_{j}^{s}$ values, indicating the power each BTS uses on each sub-band. The per channel signal to interference and noise ratio (SINR) between user $u$ and BTS $j$, on sub-band $s \in \mathcal{S}$, is defined as:

$$
\gamma_{u, j}^{s}=\frac{\frac{P_{j}^{s}}{k} \cdot G_{u, j}}{\mathcal{N}_{0}+\sum_{h \in \mathcal{J}, h \neq j} \frac{P_{h}^{s}}{k} \cdot G_{u, h}}
$$

where $\mathcal{N}_{0}$ is the additive white Gaussian noise power, $G_{u, j}$ is the channel gain between user $u$ and BTS $j$, that accounts for the path loss $\Gamma_{u, j}$, shadow fading, antenna gain $G^{a}$, and equipment/penetration losses $L$.

We denote by $R_{u, j}^{s}$ the rate seen by user $u$ from BTS $j$ on sub-band $s . R_{u, j}^{s}=k \cdot f\left(\gamma_{u, j}^{s}\right)$, where $f($.$) is the rate function$ mapping the per channel SINR to the data rate. In practice, $f($.$) is a discrete function of the SINR (see Fig. 1), which$ 
makes the analysis more difficult. In our model, we will use a continuous approximation for $f($.$) , given in Eq. (2), where$ $R_{\max }$ is the maximum achievable rate set by the practical modulation and coding scheme, and $\eta$ and $\Delta$ are constant parameters. This is very similar to the upper bounding function proposed in [29].

$$
R_{u, j}^{s}=k \cdot \min \left(\eta\left(\gamma_{u, j}^{s}\right)^{\Delta}, R_{\max }\right)
$$

For the LTE discrete function given in Table III in [34], the values of $\Delta$ and $\eta$ are computed to minimize the area between the two curves while making sure that the continuous curve is always an upper bound. The values are: $\Delta=0.43$ and $\eta=0.168$.

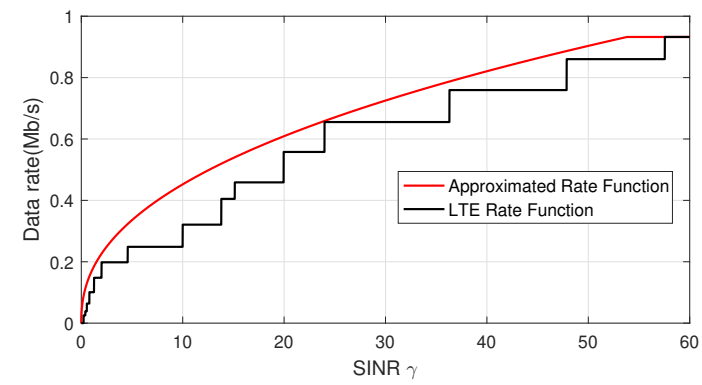

Fig. 1. The original MCS rate function $f(\gamma)$ and the upper bound we constitute

Given a power map, the scheduling is local (i.e., independent on each BTS) and allocates the sub-bands to users in a time-division fashion so as to maximize a proportionally fair objective function. Specifically, let $\alpha_{u, j}^{s}$ be the proportion of time the channels in sub-band $s$ are allocated to user $u$ in cell $j$. Then, the actual throughput $\lambda_{u}$ of user $u$ is:

$$
\lambda_{u}=\sum_{s \in \mathcal{S}} \alpha_{u, j}^{s} \cdot R_{u, j}^{s} \quad, \forall u \in \mathcal{U}_{j}
$$

Finally, we consider that, under a proportional fairness criteria, the metric to use to measure efficiency and fairness is the geometric mean (GM) throughput [34], written as:

$$
\Lambda=\left(\prod_{u \in \mathcal{U}} \lambda_{u}\right)^{1 /|\mathcal{U}|}
$$

\section{SELECTION OF A ROBUST POWER MAP}

Our goal is to find a robust power map for a given network topology, i.e., a power map that works well independently of the actual network state (e.g., the varying number of concurrent users and their association). In this power map, each BTS can transmit on each sub-band, with a different power level. Such a power map will be computed offline before network deployment, with only the knowledge of the network topology. Then, it is fixed and followed by the BTSs throughout the network operation. This power map is computed with no prior information on the actual user density and/or distribution in the network.

\section{A. Problem formulation}

In order to select a robust power map for a given network characterized by the set of BTSs $\mathcal{J}$ and the number of available channels $M$, we generate a priori a set of realizations, referred to hereafter as calibration realizations. Let $\Omega$ be the set of calibration realizations. Each realization $\omega$ corresponds to a random deployment of users in the network, and is characterized by the number of users $N_{\omega}$, the $\mathcal{U}_{j}$ for all $j \in \mathcal{J}$ and the channel gains for each pair of BTS and user.

In the following, we formulate the problem $\mathcal{P}_{1}$ that takes a given topology and a set of calibration realizations as input, and returns one power map as output. Aiming at a proportional fair objective function, the objective function in $\mathcal{P}_{1}$ (Eq. (5)) maximizes the arithmetic mean, over all the calibration realizations $\omega$, of the corresponding GM throughput $\Lambda(\omega)$ (Eq. (6)).

In constraint (7) and constraint (8), the SINR $\gamma_{u, j}^{s}(\omega)$ and the rate $R_{u, j}^{s}(\omega)$ on sub-band $s$ between BTS $j$ and user $u \in$ $\mathcal{U}_{j}$ are computed, for each realization $w$. In constraint (10), the throughput $\lambda_{u}(\omega)$ of each user in realization $\omega$ is computed. Constraint (11) ensures that the total power used by a BTS on all the sub-bands does not exceed the maximum transmission power of a BTS, $P_{j}$. Constraint (12) represents the scheduling constraint, where the sum of the time proportions allocated for all the users of a BTS on a single sub-band cannot exceed 1 , where $\alpha_{u, j}^{s}(\omega)$ is the proportion of time allocated to user $u \in \mathcal{U}_{j}$ in sub-band $s$ on realization $\omega$. Finally, constraint (13) states that all the variables in $\mathcal{P}_{1}$ are continuous and positive.

$$
\begin{aligned}
& \mathcal{P}_{\mathbf{1}}: \max _{\substack{P_{j}^{s}, \lambda_{u}(\omega), \gamma_{u, j}^{s}(\omega), R_{u, j}^{s}(\omega), \alpha_{u, j}^{s}(\omega)}} \mathcal{Z} \\
& \mathcal{Z}=\frac{\sum_{\omega \in \Omega}\left(\prod_{u \in \mathcal{U}(\omega)} \lambda_{u}(\omega)\right)^{\frac{1}{|\mathcal{U}(\omega)|}}}{|\Omega|} \\
& \gamma_{u, j}^{s}(\omega)=\frac{P_{j}^{s} \cdot G_{u, j}(\omega)}{k\left(\mathcal{N}_{0}+\sum_{h \in \mathcal{J}, h \neq j} \frac{P_{h}^{s}}{k} \cdot G_{u, h}(\omega)\right)}, \\
& \forall j \in \mathcal{J}, \forall u \in \mathcal{U}_{j}(\omega), \forall s \in \mathcal{S}, \forall \omega \in \Omega \\
& R_{u, j}^{s}(\omega)=k \cdot \eta \cdot\left(\gamma_{u, j}^{s}(\omega)\right)^{\Delta} \text {, } \\
& \forall j \in \mathcal{J}, \forall u \in \mathcal{U}_{j}(\omega), \forall s \in \mathcal{S}, \forall \omega \in \Omega \\
& R_{u, j}^{s}(\omega) \leq k \cdot R_{\max } \text {, } \\
& \forall j \in \mathcal{J}, \forall u \in \mathcal{U}_{j}(\omega), \forall s \in \mathcal{S}, \forall \omega \in \Omega \\
& \lambda_{u}(\omega)=\sum_{s \in \mathcal{S}} \alpha_{u, j}^{s}(\omega) \cdot R_{u, j}^{s}(\omega), \\
& \forall u \in \mathcal{U}_{j}(\omega), \forall j \in \mathcal{J}, \forall \omega \in \Omega \\
& \sum_{s \in \mathcal{S}} P_{j}^{s} \leq P_{j}, \forall j \in \mathcal{J} \\
& \sum_{u \in \mathcal{U}_{j}(\omega)} \alpha_{u, j}^{s}(\omega) \leq 1, \forall s \in \mathcal{S}, \forall j \in \mathcal{J}, \forall \omega \in \Omega \\
& P_{j}^{s} \geq 0, \lambda_{u}(\omega) \geq 0, \gamma_{u, j}^{s}(\omega) \geq 0, \\
& R_{u, j}^{s}(\omega) \geq 0, \alpha_{u, j}^{s}(\omega) \geq 0
\end{aligned}
$$




\section{B. Solving problem $\mathcal{P}_{1}$}

Problem $\mathcal{P} 1$, as formulated in Sec. IV-A, is a non-convex problem with non-linear constraints. In order to solve it, $\mathcal{P} 1$ is first transformed to belong to the class of nonlinear optimization called signomial programming problems, and more specifically to complementary geometric programming (GP) problems [35]. GP is a class of nonlinear, non-convex optimization problems with many useful theoretical and computational properties. A GP can be easily turned into a convex optimization problem through a logarithmic change of variable, and a global optimum can always be efficiently computed [36]. In a GP problem, all inequality constraints should be of the form $g(x)<1$, with $g(x)$ a posynomial ${ }^{1}$. In a complementary GP, a constraint can be of the form $g_{1}(x) / g_{2}(x)<1$, with both $g_{1}(x)$ and $g_{2}(x)$ being posynomials, even though the ratio of two posynomials is not a posynomial. A complementary GP can be turned into a series of GPs that can be solved by further transforming them into convex problems [35].

1) Transformation into a complementary geometric program: Problem $\mathcal{P}_{1}$ can be easily transformed into a complementary GP, by simply re-writing the constraints to match the typical structure of a complementary GP [36]. First, since we are solving a maximization problem, we replace the equality constraints with inequality constraints, which does not affect the optimal point of the problem. Then, we re-write the constraints as upper-bounded posynomials, or, when needed, ratios between two posynomials. The complementary GP problem, denoted $\mathcal{P}_{1}^{*}$, is presented in the Appendix A.

2) Single condensation method for GP: Complementary GP problems can be solved with the single condensation method, in which they are converted into a series of GP problems using an iterative algorithm [35]. To transform the complementary GP into a GP, the constraints that are not posynomials (i.e., a ratio of posynomials) should be transformed into posynomials. This is done by approximating the denominator with a monomial, while leaving the numerator as a posynomial. In general, for a posynomial $g(x)=\sum_{i} h_{i}(x)$, at a given point $x=y$, the monomial approximation $\widetilde{g}_{y}(x)$ is written as:

$$
\widetilde{g}_{y}(x)=\prod_{i}\left(\frac{h_{i}(x)}{\beta_{i}(y)}\right)^{\beta_{i}(y)}
$$

where:

$$
\beta_{i}(y)=\frac{h_{i}(y)}{g(y)}
$$

In order to find $y$, the point around which $g(x) \approx \widetilde{g}_{y}(x)$, an iterative approach is used, whose details are given in Algorithm 1. Note that in the algorithm, $\mathbf{s}^{(\mathbf{i})}$ corresponds to the point, around which we perform the approximation in the $i^{t h}$ iteration. That fixed point is simply a vector consisting of all the variables in the problem. The variable $x$ in lines 2 and 3 of Alg. 1 are the variables used in Eqs. (6) and (10), which we approximate as monomials.

\footnotetext{
${ }^{1}$ A posynomial is a sum of monomials, of the form $g(x)=\sum_{k=1}^{K} d_{k} x_{1}^{a_{k}^{(1)}} x_{2}^{a_{k}^{(2)}} \ldots x_{n}^{a_{k}^{(n)}}$
}

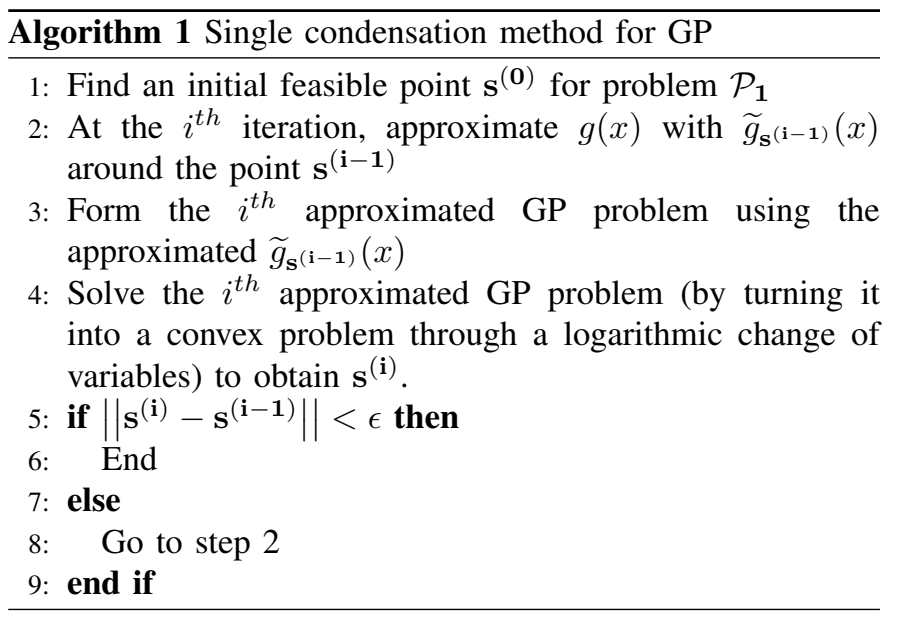

In our case, when re-written in the complementary GP form, two constraints from $\mathcal{P} 1$ become upper-bounded ratios of posynomials: Eq. (6) and Eq. (10). Hence, those two constraints should be approximated by posynomials at step 2 of Algorithm 1. For each iteration $i$, Eq. (6) is approximated by Eq. (16):

$$
\begin{array}{r}
\frac{\mathcal{Z} \cdot|\Omega|}{\prod_{w \in \Omega}\left(\frac{\left(\prod_{u \in \mathcal{U}(\omega)} \lambda_{u}(\omega)\right)^{\frac{1}{\mathcal{U}(\omega)}}}{q^{i}(\omega)}\right)^{q^{i}(\omega)}} \leq 1, \\
\forall j \in \mathcal{J}, \forall u \in \mathcal{U}_{j}(\omega)
\end{array}
$$

where $q^{i}(\omega)$ is computed as shown in Eq. (17), from the values of $\lambda_{u}^{i-1}(\omega)$ obtained from the solution at iteration $i-1$.

$$
q^{i}(\omega)=\frac{\left(\prod_{u \in \mathcal{U}(\omega)} \lambda_{u}^{i-1}(\omega)\right)^{\frac{1}{|\mathcal{U}(\omega)|}}}{\sum_{y \in \Omega}\left(\prod_{u \in \mathcal{U}(y)} \lambda_{u}^{i-1}(y)\right)^{\frac{1}{|\mathcal{U}(y)|}}}
$$

Similarly, Eq. (10) is replaced by Eq. (18), where we omit the variable $\omega$ for brevity:

$$
\frac{\lambda_{u}}{\prod_{s \in \mathcal{S}}\left(\frac{\alpha_{u, j}^{s} \cdot R_{u, j}^{s}}{\theta_{u, j}^{s, i}}\right)^{\theta_{u, j}^{s, i}}} \leq 1, \forall j \in \mathcal{J}, \forall u \in \mathcal{U}_{j}
$$

The new exponent $\theta_{u, j}^{s, i}$ is computed as shown in Eq. (19), from the values of $\alpha_{u, j}^{s, i-1}$ and $R_{u, j}^{s, i-1}$, obtained from the solution at iteration $i-1$.

$$
\theta_{u, j}^{s, i}=\frac{\alpha_{u, j}^{s, i-1} \cdot R_{u, j}^{s, i-1}}{\sum_{l \in \mathcal{S}} \alpha_{u, j}^{l, i-1} \cdot R_{u, j}^{l, i-1}}
$$

Following these approximations, the obtained problem is a non-linear, non-convex GP. At step 4 in Algorithm 1, this problem can be solved efficiently by turning it into the following convex problem, denoted $\mathcal{P}_{2}$, through a logarithmic change of variables. Let $l_{u}(\omega)=\log \lambda_{u}(\omega), z=\log (\mathcal{Z})$, $p_{j}^{s}=\log P_{j}^{s}, a_{u, j}^{s}(\omega)=\log \alpha_{u, j}^{s}(\omega), y_{u, j}^{s}(\omega)=\log \gamma_{u, j}^{s}(\omega)$, $r_{u, j}^{s}(\omega)=\log R_{u, j}^{s}(\omega)$, and $o_{u, j}^{s}(\omega)=\log \theta_{u, j}^{s}(\omega)$. 


$$
\begin{aligned}
& \mathcal{P}_{\mathbf{2}}:_{p_{j}^{s}(\omega), l_{u}(\omega), y_{u, j}^{s}(\omega), r_{u, j}^{s}(\omega), a_{u, j}^{s}(\omega)} z \\
& z+\log |\Omega|-\sum_{w \in \Omega} q(\omega)\left(\sum_{u \in \mathcal{U}(\omega)} \frac{l_{u}(\omega)}{|\mathcal{U}(\omega)|}+\log q(\omega)\right) \leq 0
\end{aligned}
$$

$$
\log \left(\frac{e^{\left(y_{u, j}^{s}(\omega)-p_{j}^{s}\right)}\left(k \cdot \mathcal{N}_{0}+\sum_{\substack{h \in \mathcal{J} \\ h \neq j}} G_{u, h}(\omega) \cdot e^{p_{h}^{s}}\right)}{G_{u, j}(\omega)}\right) \leq 0,
$$$$
\forall j \in \mathcal{J}, \forall u \in \mathcal{U}_{j}(\omega), \forall s \in \mathcal{S}, \forall w \in \Omega
$$

$r_{u, j}^{s}(\omega)-\Delta \cdot y_{u, j}^{s}(\omega)-\log k-\log x \leq 0$,

$\forall j \in \mathcal{J}, \forall u \in \mathcal{U}_{j}(\omega), \forall s \in \mathcal{S}, \forall w \in \Omega$

$$
r_{u, j}^{s}(\omega)-\log k-\log R_{\max } \leq 0 \text {, }
$$$$
\forall j \in \mathcal{J}, \forall u \in \mathcal{U}_{j}(\omega), \forall s \in \mathcal{S}, \forall w \in \Omega
$$

$$
l_{u}(\omega)+\sum_{s \in \mathcal{S}} \theta_{u, j}^{s}(\omega)\left(o_{u, j}^{s}(\omega)-a_{u, j}^{s}(\omega)-r_{u, j}^{s}(\omega)\right) \text {, }
$$$$
\forall u \in \mathcal{U}_{j}(\omega), \forall j \in \mathcal{J}, \forall w \in \Omega
$$

$$
\log \left(\sum_{s \in \mathcal{S}} e^{p_{j}^{s}}\right)-\log P_{j} \leq 0, \forall j \in \mathcal{J}
$$

$\log \left(\sum_{u \in \mathcal{U}_{j}(\omega)} e^{a_{u, j}^{s}(\omega)}\right) \leq 0, \forall s \in \mathcal{S}, \forall j \in \mathcal{J}, \forall w \in \Omega$

$p_{j}^{s} \geq \delta, t \geq \delta, l_{u}(\omega) \geq \delta, y_{u, j}^{s}(\omega) \geq \delta$,

$r_{u, j}^{s}(\omega) \geq \delta, a_{u, j}^{s}(\omega) \geq \delta$

\section{Numerical Results for the Static Setting}

In this section, we will give the numerical results for a static setting, where we evaluate the performance of the proposed method over a large number of independent snapshots and study the impact of the power map parameters. We will first consider homogeneous networks, followed by heterogeneous networks. We will evaluate the performance of the proposed method in a dynamic setting in the next section.

\section{A. Homogeneous networks}

We begin with homogeneous networks, where all the BTSs are macro BTSs with the same high power budget. For this case, we will first show that the proposed method works well using a simple example and then evaluate the impact of each parameter on the network performance. We will finally compare the performance of SFR with the performance of our power map method.

1) Results on a toy scenario: Let us first consider the homogeneous network topology in Fig. 2, with $|\mathcal{J}|=5$ BTSs, randomly deployed in an area of 1 unit square. We consider a system with $M=120$ channels, $T=10$ subframes in a frame, and a distance-based path loss model for an urban setting, such that $\Gamma_{u, j}=128.1+37.6 \cdot \log \left(D_{u, j} / 1000\right) \mathrm{dB} \quad$ (if $D_{u, j}>35$ m) [37]. We set the power budget of all BTSs to $P_{j}=46$ $\mathrm{dBm}$, the antenna gain of the BTS to $G^{a}=10 \mathrm{~dB}$, equipment losses to $L=20 \mathrm{~dB}$, noise power to $\mathcal{N}_{0}=-121 \mathrm{dBm}$, and we model shadow fading through the normal distribution with zero mean and standard deviation $\sigma=8 \mathrm{~dB}$. For the rate function in Eq. (2), we set the parameters $\eta=0.168, \Delta=0.43$, and $R_{\max }=932.4 \mathrm{~Kb} / \mathrm{s}$, approximating the rate function in a LTE system. Throughout the paper, all computations are done using the open-source solver Bonmin [38], on a server with Intel Xeon CPU E5-2697 v2 @ 2.70GHz.

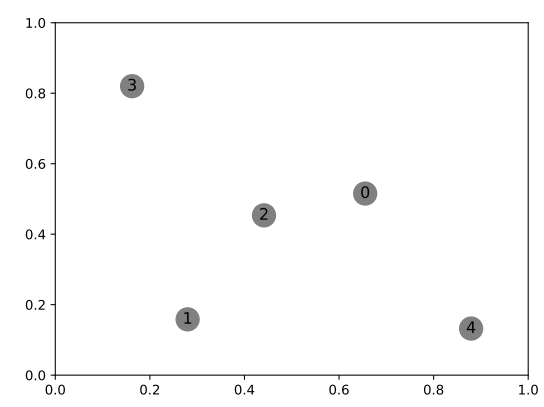

Fig. 2. Network topology with 5 BTSs.

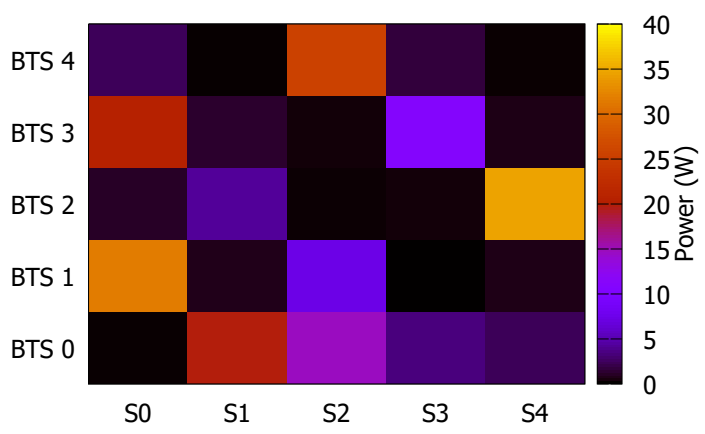

Fig. 3. Power map for the network in Fig. 2, obtained for a certain $\Omega$ with $|\Omega|=10, N_{\omega}=100$ users and $b=5$ sub-bands. For each BTS, one sub-band is used with high power and the others are used with lower power. Distant BTSs such as BTSs 1 and 3 can use the high power on the same band due to lower interference.

In the following, we show a numerical example of a power map computation, for a given set of parameters, i.e., a value of $b$, the number of bands, and a set of calibration realizations. Once found, we evaluate the power map performance on another set of realizations (called the test set) by computing the achieved GM throughput for each realization and comparing it to the one obtained with a classical equal power/fixed frequency reuse scheme. The impact of all the input parameters in the power map computation are studied in detail next.

a) Finding the power map: Following all the steps presented in Sec. IV allows us to solve problem $\mathcal{P}_{1}$, and get a power map with $b$ sub-bands, for a given topology, based on a set of calibration realizations $\Omega$. For example, we consider a set $\Omega$ of 10 calibration realizations (i.e., $|\Omega|=10$ ). In each realization $\omega$, we randomly distribute $N_{\omega}=100$ users, and assume that a user associates to the BTS that yields the highest channel gain. We set $b=5$ sub-bands. Given $\Omega$, and $b=5$, a representation of the power map obtained using Algorithm 1 is shown in Fig. 3. 
We notice that, for each BTS, there is at least one sub-band on which the power is relatively higher than on the other sub-bands. This sub-band is generally different for each BTS, meaning two BTSs rarely transmit with a high power on the same channels. On the other hand, while a BTS transmits with high power on one sub-band, it also transmits on almost all the other sub-bands, but with relatively lower powers. What we observe is the following: all BTSs use all the channels, and allocate few channels with high power (low interference) to users with relatively bad channel conditions (e.g., farthest from BTS), and plenty of channels with lower power to users with better channel conditions (e.g., closest to the BTS). Some BTSs use very small power on some sub-bands, which creates almost no interference to the neighboring cells. However, such sub-bands are still useful since good rates can be offered to users with high channel gains.

b) Testing the power map: In this section, we test the performance of the obtained power map on a set of test realizations, capturing different user distributions in the network. We continue to assume that a user associates with the BTS offering the best channel gain. For each test realization, since the transmit powers of the BTSs on each sub-band $P_{j}^{s}$ are known, the corresponding inter-BTS interference, SINR (Eq. (1)), and rate (Eq. (2)) can be directly computed. The scheduling can be done locally at each BTS, for all the users associated to it, independently of the other BTSs, by respecting the computed power map. The scheduling assigns for each user $u$, associated to BTS $j$, the proportion of time, $\alpha_{u, j}^{s}$, during which all the channels of sub-band $s$ are allocated to $u$. We consider that users of each BTS are optimally scheduled (we will propose an online practical scheduler in Sec. VI), with the objective of maximizing the proportional fair objective function in a cell. The local scheduler in cell $j$ is formulated below, where the $R_{u, j}^{s}$ are computed beforehand, given the power map:

$$
\begin{aligned}
& \max _{\lambda_{u}, \alpha_{u, j}^{s}} \sum_{u \in \mathcal{U}_{j}} \log \left(\lambda_{u}\right) \\
& \lambda_{u}=\sum_{s \in \mathcal{S}} \alpha_{u, j}^{s} \cdot R_{u, j}^{s}, \forall u \in \mathcal{U}_{j} \\
& \sum_{u \in \mathcal{U}_{j}} \alpha_{u, j}^{s} \leq 1, \forall s \in \mathcal{S} \\
& \lambda_{u} \geq 0, \alpha_{u, j}^{s} \geq 0
\end{aligned}
$$

This is a simple problem to solve and given the throughput of each user in each cell, the GM throughput of the test realization is computed according to Eq. (4).

Let $\Pi$ be the set of test realizations, with each realization $\pi \in \Pi$ corresponding to a random deployment of users in the network, $\mathcal{U}(\pi)$, all with the same number of users $N_{\Pi}$. For each of the realizations in $\Pi$, we compute the throughput geometric mean, $\Lambda_{P M}$, obtained when the computed power map is used by the BTSs. We remind the reader that the power map was computed independently of $\Pi$, using a different set of realizations $\Omega$. For comparison, we also compute, for the same realizations, the GM throughput, $\Lambda_{E P-R_{r}}$, obtained when a classical fixed frequency reuse scheme, with a reuse factor $r$, is used along with a round robin scheduler and equal power on each subchannel.

Fig. 4 shows the values of the computed throughput geometric means, $\Lambda_{P M}, \Lambda_{E P-R 1}$, and $\Lambda_{E P-R 3}$, obtained with the power map in Fig. 3, an equal power/reuse 1 scheme, and an equal power/reuse 3 scheme, respectively. The results are averaged on $|\Pi|=100$ snapshots, with $N_{\pi}=100$ users in each. The confidence intervals are at $95 \%$. It is clear in Fig. 4 that our power map outperforms the classical equal power maps, for both reuse 1 and reuse 3 schemes. Indeed, we notice a gain of $42 \%$ with respect to equal power/reuse 1 , and a gain of $64 \%$ with respect to equal power/reuse 3 .

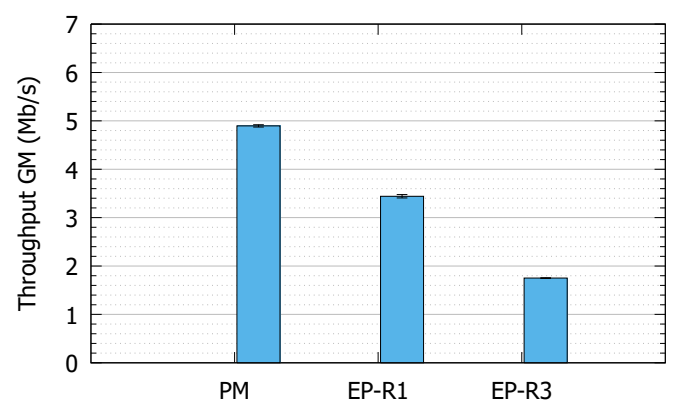

Fig. 4. Comparison of the throughput geometric mean with the power map in Fig. 3 (PM), a classical equal power with reuse 1 scheme (EP-R1), and a classical equal power with reuse 3 scheme (EP-R3).

For the same snapshots in $\Pi$, we show in Fig. 5 the cumulative distribution function (CDF) of the throughputs of individual users, i.e., the CDF of $\lambda_{u}, \forall u \in \mathcal{U}(\pi), \forall \pi \in \Pi$. It is clear in Fig. 5 that the user throughput values are higher with our map, and the range to which they belong is wider. Indeed, while the achieved throughput per user ranges between 0.45 and $16 \mathrm{Mb} / \mathrm{s}$ with the equal power/reuse 1 scheme, it is between 0.95 and $25 \mathrm{Mb} / \mathrm{s}$ with our power map. This shows that the gain in the GM throughput, seen in Fig. 4, is due to an increase in all the users' throughputs in the network when the BTSs follow our map.

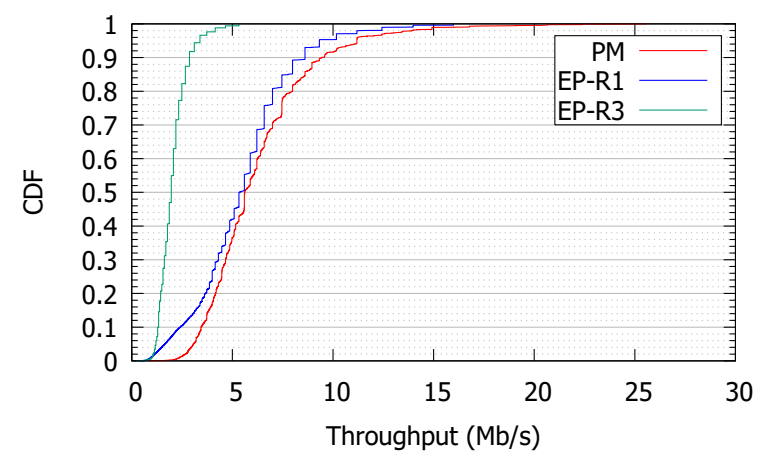

Fig. 5. CDF of the users' individual throughputs, with the power map in Fig. 3 (PM), a classical equal power with reuse 1 scheme (EP-R1), and a classical equal power with reuse 3 scheme (EP-R3).

Note that the BTSs do not coordinate with each other during their operation. The power map computation is done offline and all the BTSs need to know is the power map used by each BTS. 
2) Effects of the algorithm parameters: Computing the power map depends on several tunable parameters, raising some questions on its performance with respect to those parameters. First of all, each power map depends on a particular network, i.e. a given topology of a precise number of BTSs $|\mathcal{J}|$. Does the procedure shown in Sec. IV still holds regardless of the number of BTSs in the network and their topology? Second, the power map corresponds to a given number of sub-bands $b$, which determines the number of subchannel sets the frequency band is divided into. How many sub-bands are sufficient for the power map to perform? Third, finding the power map is based on two given parameters: the number of calibration realizations $|\Omega|$, and the number of users in each calibration realization $N_{\omega}$. How many calibration realizations are enough to obtain a robust power map? How does the obtained power map with a given $N_{\omega}$ perform in networks with a varying number of users? In the following, we answer those questions by varying each of those parameters, and studying their effect on the power map performance and give useful insights on how to set those parameters.

a) Number of sub-bands: As detailed earlier, the frequency band is divided into $b$ sub-bands, and each BTS transmits on the channels of each sub-band with a different power level. In other words, the number of sub-bands $b$ also corresponds to the number of possibly different power levels of a BTS. In the following, we test different values of $b$ to observe its effect on the power map performance, and to estimate how many sub-bands (or power levels) are considered to be enough.

For the network topology in Fig. 2 with $|\mathcal{J}|=5$ BTSs, we fix $|\Omega|=10$ calibration realizations, and $N_{\omega}=100$ users in all calibration realizations $\omega$, and we vary the number of sub-bands $b$. For each value of $b$, we first find the corresponding power map. Then, we compute the GM throughput, $\Lambda_{P M}(b)$, achieved with the resulting power map in $|\Pi|=100$ test realizations, with $N_{\pi}=100$ randomly distributed users in each realization. For the same realizations, we compute the GM throughput, $\Lambda_{E P-R 1}$, achieved with a classical equal power/reuse 1 scheme (we omit the scheme with reuse 3 since it performs much worse than reuse 1).

We show the results in Fig. 6. We note that, by simply dividing the channels into $b=2$ sub-bands, i.e., each BTS transmits with 2 power levels, one for each sub-band, a gain of $27 \%$ in GM throughput can be achieved with respect to classical equal power/reuse 1 scheme. This gain can go up to $49 \%$ with $b=10$ sub-bands. Nevertheless, increasing the number of sub-bands $b$, also increases the size of problem $\mathcal{P}_{1}$, which increases the corresponding solving time, due to the convergence time of Algorithm 1, although, since it is an offline computation that is done before deployment, this is not necessarily a major issue. Note that the gain in capacity, i.e., in the arithmetic mean throughput, not shown in the figure, is $18 \%$ for $b=2$ sub-bands and $30 \%$ for $b=10$ sub-bands.

b) Number of calibration realizations: Another critical parameter when computing the power map is the number of calibration realizations $|\Omega|$. Indeed, the objective function in $\mathcal{P}_{1}$ (Sec. IV-A) is a maximization of the average of the throughput geometric means over all the calibration realizations. Intuitively, the more calibration realizations are

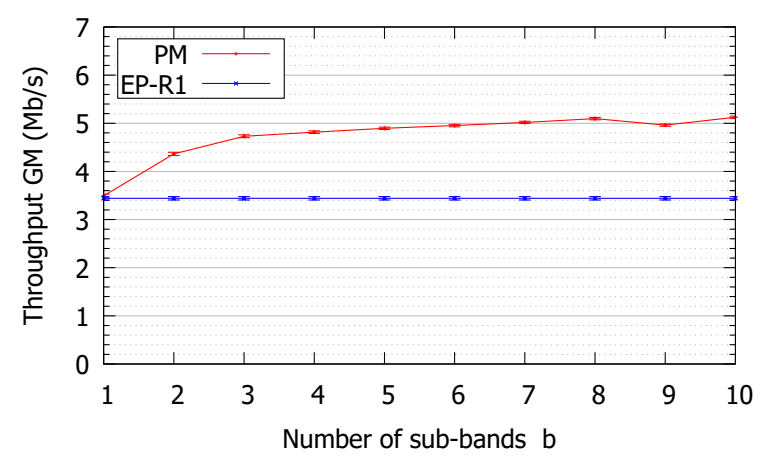

Fig. 6. Throughput GM as a function of the number of sub-bands $b$, tested on $|\Pi|=100$ test realizations, with $N_{\pi}=100$ users in each realization, with a power map obtained for $|\Omega|=10$ calibration realizations, $N_{\omega}=100$.

used, the more robust the computed power map. In the following, we test different values of $|\Omega|$ to observe its effect on the power map robustness, in order to find a suitable value.

For the network topology in Fig. 2, we vary the number of calibration realizations $|\Omega|$, while fixing $N_{\omega}=100$ users in each calibration realization $\omega$, and $b=5$ sub-bands. For $|\Pi|=100$ test realizations, with $N_{\pi}=100$ users in each, we compute the GM throughput $\Lambda_{P M}(|\Omega|)$ achieved with the resulting power map for each value of $|\Omega|$, and compare it to the GM throughput $\Lambda_{E P-R 1}$ achieved with a classical equal power/reuse 1 scheme (with a performance that does not depend on $|\Omega|$ ).

Results in Fig. 7 show that only a few calibration realizations are enough in order to get a robust power map. Indeed, with only $|\Omega|=5$ calibration realizations, the gain in throughput geometric mean, with respect to the classical equal power/reuse 1 scheme, is $42 \%$.

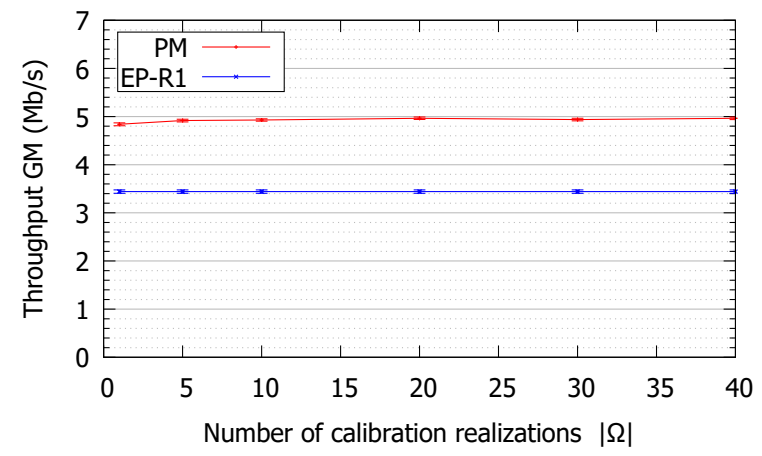

Fig. 7. Throughput geometric mean function of the number of calibration realizations $|\Omega|$ used to obtain the power map, for $b=5$ sub-bands, and $N_{\omega}=100$ users, tested on $|\Pi|=100$ test realizations, with $N_{\pi}=100$ users in each test realization.

c) Number of users: The power map is obtained based on a set of calibration realizations $\Omega$, each of them with a random distribution of $N_{\omega}$ users, and it is tested on a set of different realizations $\Pi$, each having $N_{\Pi}$ users. In the following, we check whether a power map obtained based on calibration realizations with $N_{\omega}$ users can still be used in networks with $N_{\Pi}$ users, even when $N_{\Pi} \neq N_{\omega}$. In other words, we verify the robustness of the power map with respect to the number of users in the network. 
For the network topology in Fig. 2, we fix $|\Omega|=10$ calibration realizations, and $b=5$ sub-bands. We vary the number of users $N_{\omega}$ in the calibration realizations, and find the corresponding power map for each $N_{\omega}$. Then, we take 4 sets of $|\Pi|=100$ test realizations each. Each of the sets has a different number of users, i.e., $N_{\Pi} \in\{25,50,100,200\}$. We test the power maps obtained for each value of $N_{\omega}$, on the different test realization sets, i.e., we compute the GM throughput $\Lambda_{P M}\left(N_{\omega}, N_{\Pi}\right)$ for all the combinations $\left(N_{\omega}, N_{\Pi}\right)$. We compare them to the GM throughput $\Lambda_{E P-R 1}\left(N_{\Pi}\right)$ achieved with a classical EP-R1 scheme for the different values of $N_{\Pi}$.

Results in Fig. 8 show that, even when $N_{\Pi} \neq N_{\omega}$, the throughput GM is not affected. This is why the different curves corresponding to different values of $N_{\omega}$ are actually indistinguishable, independently of the value of $N_{\Pi}$. Indeed, there is practically no difference in the outcome when a power map obtained with calibration realizations with $N_{\omega}$ users is tested on test realizations with $N_{\Pi} \neq N_{\omega}$ users (but following the same distribution). This means that, even with a lack of information on the number of users in a network during the deployment phase, the initial power map can be obtained based on calibration realizations with a reasonable value of $N_{\omega}$, and still be performing well, regardless of the actual number of users in the network. Moreover, this is an indicator of the power map robustness facing drops or increases in the number of users throughout the network deployment.

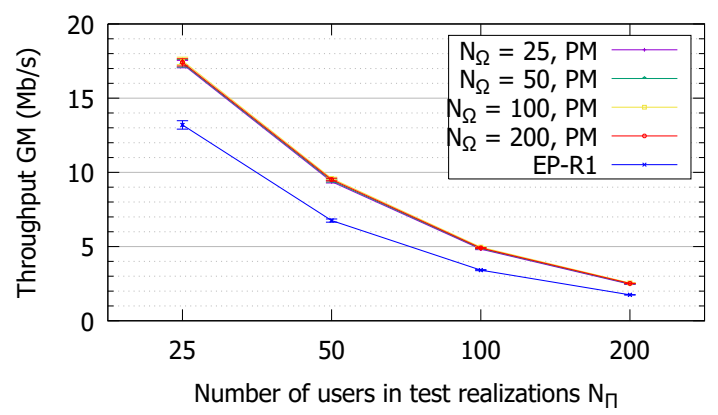

Fig. 8. Throughput geometric mean function of the number of users in test realizations $N_{\pi}$, for different values of the number of users in calibration realizations $N_{\omega}$, for $b=5$ sub-bands, and $|\Pi|=100$ test realizations.

d) Network topology: All the previous results were obtained for the particular topology of 5 BTSs shown in Fig. 2. In order to verify and generalize the results, we repeated the previous study on a multitude of topologies, with different numbers of BTSs. All the results are consistent with the previous ones, and all the conclusions hold, regardless of the network topology or the number of BTSs in question.

We show an example in Fig. 9 of a network topology with $|\mathcal{J}|=15$ BTSs. Fig. 10 shows the power map computed for this topology, with the following parameters: $b=5$ sub-bands, $|\Omega|=10$ calibration realizations, and $N_{\omega}=150$ users in each calibration realization. We notice that, on each sub-band, there is at least one BTS that transmits with a significantly higher power than the others. Most of the BTSs, however, do not use their full power. Moreover, even though most of the BTSs transmit on several sub-bands, there is at least one sub-band per BTS where transmission power is negligible. The

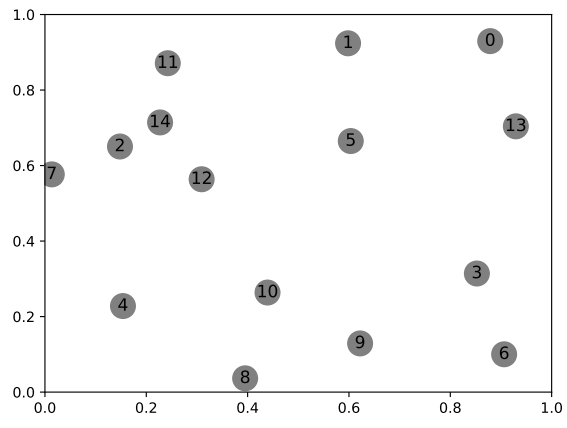

Fig. 9. Network topology with 15 BTSs.

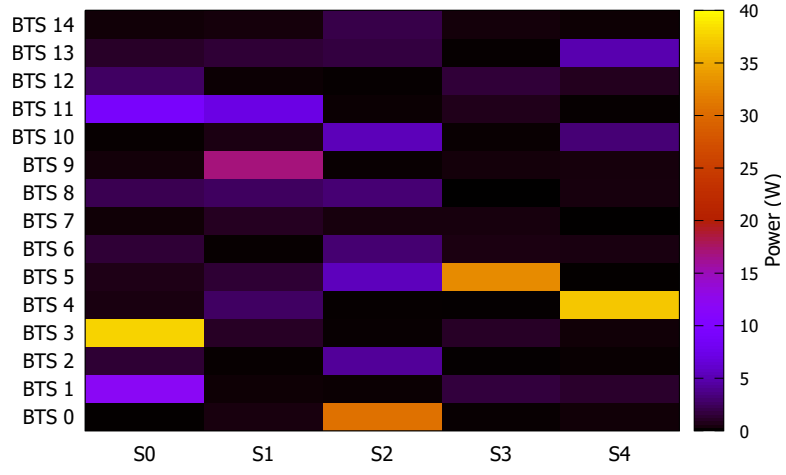

Fig. 10. Power map for the network in Fig. 9, obtained for $|\Omega|=10$ calibration realizations, $N_{\omega}=150$ users, and $b=5$ sub-bands.

distribution of transmission powers on the sub-bands can differ significantly from one BTS to another. For example, while BTS 5 has transmission powers ranging from 1 to $32 \mathrm{~W}$, on 4 sub-bands, BTS 7 transmits with powers lower than $1 \mathrm{~W}$ on all the sub-bands.

Nevertheless, we show in Fig. 11 that all BTSs are operational and serving users. Indeed, Fig. 11 shows the GM throughput achieved per BTS, i.e., the GM of the throughputs of the users associated to each BTS. These results are obtained for $|\Pi|=100$ test realizations, and $N_{\pi}=150$ users in each realization. We compare the achieved GM throughput with the power map in Fig. 10, and with an equal power/reuse 1 scheme. We note that the achieved GM throughput per BTS is inversely proportional to the number of users associated to that BTS. We notice in Fig. 11 that even BTSs with lower powers on most sub-bands, such as BTS 7, have a significant value of GM throughput. Such BTSs are mostly serving a small number of users that are close to them, i.e., with good channel conditions, and hence, do not need high transmit power.

While $b=5$ sub-bands seemed reasonable for the previous topology of 5 BTSs, we verify in the following if this is still the case for the topology with 15 BTSs. For the topology in Fig. 9, we vary $b$, and compute for each value the GM throughput, $\Lambda_{P M}(b)$, achieved with the resulting power map, for $|\Pi|=100$ test realizations, and $N_{\pi}=150$ users in each realization.

Results are shown in Fig. 12. Similarly to the observations in Fig. 6, the relative gain in the GM throughput, with respect 


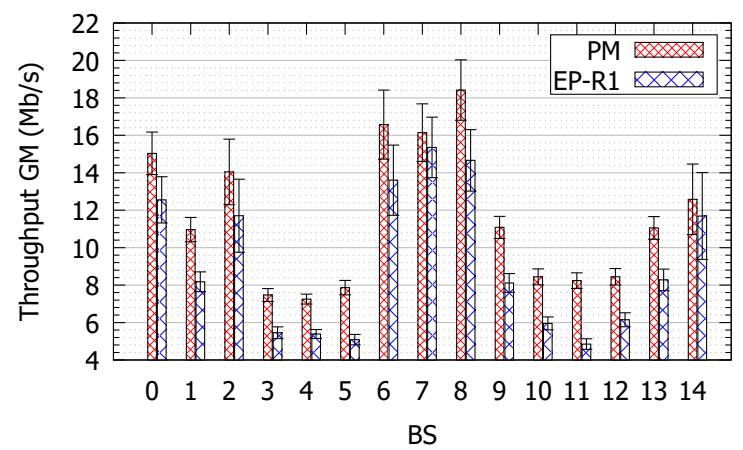

Fig. 11. The throughput GM of users associated to each BTS, with the power map in Fig. 10 (PM), and a classical equal power with reuse 1 scheme (EP-R1).

to the equal power/reuse 1 scheme, increases with the number of sub-bands. The gain is around $40 \%$ with $b=5$ sub-bands, and can go up to $46 \%$ with $b=15$ sub-bands. Thus, $b=5$ still seems to be a good compromise between good performance, and the size of problem $\mathcal{P}_{1}$, that increases with $b$.

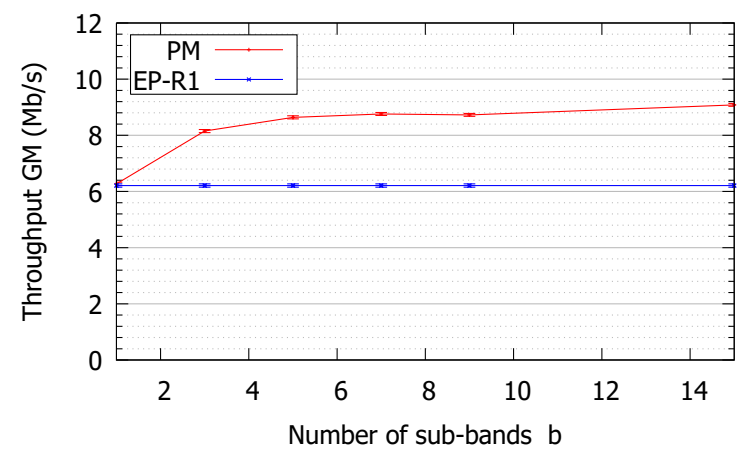

Fig. 12. Throughput geometric mean function of the number of sub-bands $b$, for $|\Omega|=10$ calibration realizations, and $N_{\omega}=150$ users, tested on $|\Pi|=150$ test realizations, with $N_{\pi}=150$ users in each test realization.

Note that we have considered flat channel gains in our computations. However, our formulation can be generalized to non-flat fading channels. Since we divide the subchannels into smaller sub-bands, it would be a reasonable assumption to have flat subchannels inside a sub-band. Then, even though the channel gains change among the sub-bands, our formulation would still work.

3) Comparison with Soft Frequency Reuse and Fractional Frequency Reuse: A special case of our power map is the soft frequency reuse (SFR) scheme [1]. SFR corresponds to a power map with three sub-bands and each BTS uses only one sub-band with a high power and the other two sub-bands with a low power. In the remaining of this paper, we will refer to this scheme as default SFR. The decision of which sub-band is used with high power by each BTS depends on the location of the BTSs, e.g., two geographically close BTSs do not use the same sub-band with high power. While SFR is a simpler way to allocate power, it is not as flexible as our power map and we show in the following that it does not perform as well as a full-fledged power map for some scenarios: $i$ ) when there are hot-spots in the network, ii) when there are multiple BTSs (more than 3) located close to each other, iii) when the power budgets of the BTSs differ significantly, e.g., in HetNets. To illustrate this, we will combine the first two cases and present some results in the following, while addressing the third case in the next subsection.

One other benchmark we will use in this subsection is fractional frequency reuse (FFR) [24]. FFR divides the subchannels into four sub-bands, where one sub-band consists of $M_{s}$ subchannels and the other three contains $M_{r}$ subchannels such that $M_{s}+3 M_{r}=M$. Similar to default SFR, the cells are divided into three reuse groups. The first $M_{s}$ subchannels are used by all the cells in a reuse one approach, with power $P^{\prime} / M_{s}$ on each subchannel, where $P^{\prime}$ is an FFR parameter. Then, the first $M_{r}$ subchannels are used by the first reuse group, the next $M_{r}$ subchannels are used by the second reuse group and the last $M_{r}$ subchannels are used by the last reuse group. The power on those subchannels will be $\frac{P-P^{\prime}}{M_{r}}$. Therefore, each cell uses $M_{s}+M_{r}$ subchannels where $M_{s}$ subchannels are used with high interference and $M_{r}$ subchannels are used with low interference. Note that, in order to parametrize an FFR scheme, we need to determine $M_{s}$ and $P^{\prime}$. In the following, we parametrize FFR using brute-force search.

Consider the network topology in Fig. 13 where each circle represents a macro BTS and the black rectangle in the middle represents a hot-spot, where the average user density is three times higher than the rest of the area. Such cases can occur in an urban scenario.

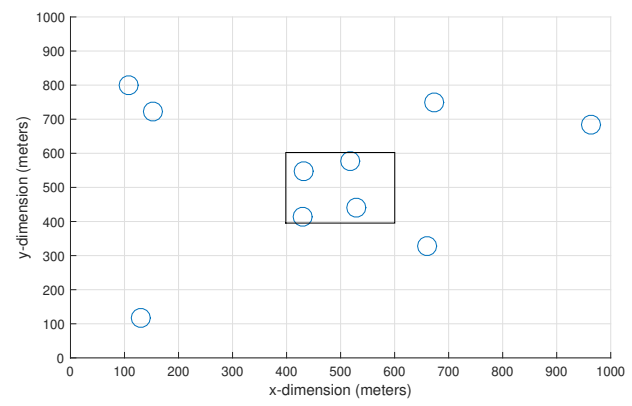

Fig. 13. An example network with 10 BTSs with a hot-spot represented in the middle of the network.

For this particular network, we compare six schemes, which are equal power/reuse 3 (EP-R3), equal power/reuse 1 (EP-R1), default SFR, fractional frequency reuse, power map with 3 bands $(b=3)$, and power map with 5 bands $(b=5)$. To compute the two power maps (one with 3 bands and one with 5 bands), we use $|\Omega|=10$ calibration realizations with $N_{\omega}=100$ users in each calibration realization. For the default SFR, we first create Voronoi cells around the BTSs and make the power assignment such that no two neighboring cells use the same sub-band with high power. To determine the exact low $(p)$ and high $(P)$ power values, we use the same $|\Omega|=10$ calibration realizations and try different ratios $P / p$ and pick the one that maximizes the arithmetic average of the GM throughput of the calibration realizations. Similarly, for FFR, we divide the cells into three groups using Voronoi cells and make a brute-force search among the values of the 
FFR parameters, which are $M_{s}$ and $P^{\prime}$. We then evaluate all schemes on $|\Pi|=200$ test realizations with $N_{\pi}=100$ users. Each user connects to the BTS that yields the highest channel gain to that user. In Fig. 14, we present the CDF of the GM throughputs over the 200 realizations for each scheme.

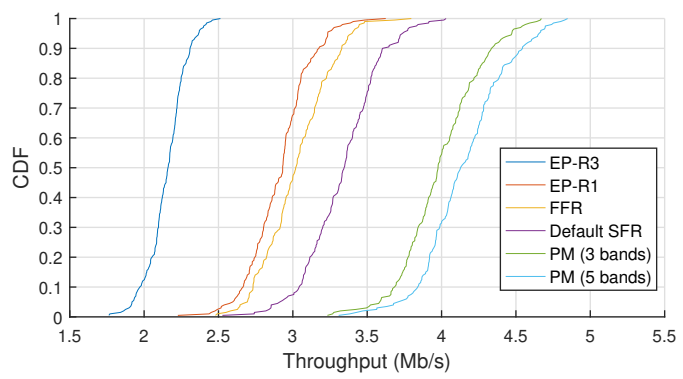

Fig. 14. CDF of the per test realization GM throughput for different schemes, for the network shown in Fig. 13, over $|\pi|=200$ test realizations with $N_{\pi}=100$ users in each test realization.

Clearly, all the schemes based on power allocation performed much better than the state of the art, which is EP-R1. For example, the average GM throughput of default SFR is $15 \%$ higher than EP-R1 and FFR is $6 \%$ better than EP-R1. The power map with 3 sub-bands performs better than the default SFR scheme: its average GM throughput is $20 \%$ higher. In terms of arithmetic mean (not shown), the difference is around $12 \%$. The power map with 5 sub-bands performs even better, as the average GM difference with the default SFR increases to $25 \%$ and the arithmetic mean difference increases to $16 \%$. The power map with 5 sub-bands performs $37 \%$ better (in average GM) than FFR parametrized optimally.

This improved performance is the consequence of the increased flexibility when setting the power levels. However, we note that the scheme remains practical and uses only offline computation, without any implication of dynamic power control.

4) Further analysis on the robustness of the power map: Next, we perform some more computations to further illustrate the robustness of the power map. Recall that we compute a power map beforehand using the calibration realizations and we test them on the test realizations. Our question in this step is: What would happen if we had computed instead the best power map for each test realization? Clearly, this is not a feasible approach in real time systems since the realizations can change very fast and adjusting the power allocation so fast is not a realistic approach. However, this can give us some insights on the robustness of our power map that we compute using the calibration realizations. If we can show that our power map performs almost as well as the case where we can instantly calculate the best power map for each realization, then our power map is robust and efficient.

Fig. 15 shows the results, where the realizations are sorted with respect to decreasing GM throughput when the best power map is computed for each test realization. The performance of our robust power map, which is computed using the calibration realizations, is very close to the performance of the best power allocation for each test realization, with an average difference of $6 \%$. Note that this

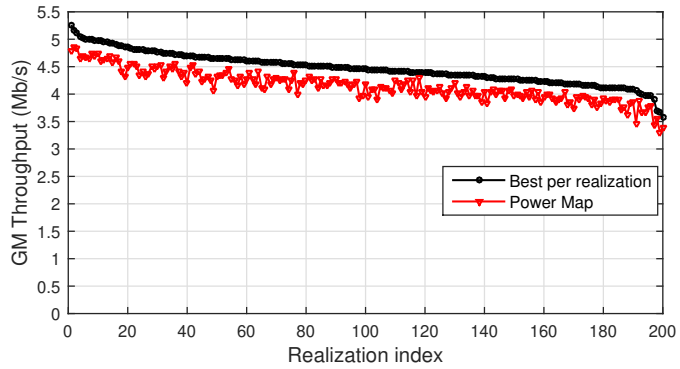

Fig. 15. Comparing the robust power map with the best power allocation per test realization for the network shown in Fig. 13, over $|\pi|=200$ test realizations with $N_{\pi}=100$ users in each test realization

small difference is because our robust power map already captures the features of many different calibration realizations and hence it works well over different test realizations.

To illustrate that a power map computed for a random realization could perform badly for other realizations and hence, the importance to use a robust power map, we perform the following experiment. Using the same network as above, we compare the performance of the best power map per realization, the power map we compute using the calibration realizations, and a number of power maps that are computed using single random realizations. Specifically, we pick five random realizations among the 200 realizations we have studied above and get the best power map for each of these five realizations. Then, we apply these power maps to all the 200 realizations and obtain the average GM throughput. Our aim is to show that the power map should be computed using many calibration realizations and the power maps that are obtained using single random realizations do not perform well enough. Table II shows the results. In the table, best corresponds to the best power map per realization, $P M$ corresponds to our power map that we compute over the calibration realizations, and the other power maps corresponds to the random power maps obtained by single random realizations.

TABLE II

GM Throughrut PERFormance OF DIFFERENT POWER MAPS FOR THE NETWORK SHOWN IN FIG. 13, OVER $|\pi|=200$ TEST REALIZATIONS WITH $N_{\pi}=100$ USERS IN EACH TEST REALIZATION

\begin{tabular}{|c|c|c|c|c|c|c|c|}
\hline & Best & PM & RM1 & RM2 & RM3 & RM4 & RM5 \\
\hline Mean & 4.43 & 4.16 & 3.70 & 3.48 & 3.59 & 3.46 & 3.79 \\
\hline Var. & 0.0812 & 0.0788 & 0.0959 & 0.0885 & 0.0997 & 0.0912 & 0.1122 \\
\hline
\end{tabular}

Table II shows that the highest average GM throughput is obtained when the best power map is selected for each realization (but this is not practical). The second best performance is obtained when our power map is used. Note that the average GM throughput of the power maps that we obtain for single random realizations are lower than the one of our power map. When their performance is relatively close, e.g., the performance of RM5, the variance of GM throughput for that random power map is quite high, i.e., much higher than the variance for our power map, which means that the performance of the power map obtained with a single random realization strictly depends on the network realization. 
5) Validation of the upper bounding rate function: Recall that we use the upper bounding rate function that is shown in Fig. 1 to simplify our calculations. In this section, we will illustrate the tightness of the results obtained with this function by showing that a feasible solution to the problem with the original piece-wise constant rate function yields very similar performance. The feasible is constructed from the solution to the upper bounding problem. To this end, we consider the network shown in Fig. 13 and we evaluate the performance of all six power allocation schemes shown in Fig. 14.

Table III shows the differences between the upper bounds and the feasible solutions.For example, a value of 3.47 in Table III means the performance with the original LTE function is $3.47 \%$ lower than the performance with the upper bounding rate function.

\section{TABLE III}

DifFERENCE (IN \%) OF GM THROUGHPUT PERFORMANCE BETWEen THE UPPER BOUNDING RATE FUNCTION AND THE LTE RATE FUNCTION FOR THE NETWORK SHOWN IN FIG. 13, OVER $|\pi|=200$ TEST REALIZATIONS, FOR DIFFERENT POWER ALLOCATION SCHEMES

\begin{tabular}{|c|c|c|c|c|c|c|}
\hline & EP-R3 & EP-R1 & FFR & SFR & PM3 & PM5 \\
\hline$N \pi=50$ & 3.87 & 4.81 & 3.57 & 3.39 & 3.31 & 3.29 \\
\hline$N \pi=100$ & 3.91 & 4.70 & 3.53 & 3.46 & 3.32 & 3.19 \\
\hline
\end{tabular}

Table III indicates that the upper bound is tight since the average GM throughput performances with the two rate functions are very close to each other, i.e., around 3-4\%. Furthermore, Table III shows that the number of users does not have much effect on the difference between the two rate functions.

\section{B. Heterogeneous networks}

Next, we evaluate our power map method in HetNets, i.e., cellular networks that comprise low-power small cells (SC), as well as high power macro cells [26]. The addition of small cells improves the throughput seen by users [34]; however, deploying a HetNet requires complex channel allocation and new user association schemes.

Chanel allocation determines which subchannels should be used by each BTS and this has to be done carefully because of the very large difference in the power budgets of the macro BTSs and the small cells. When they operate on the same channel, the users associated to the small cells would receive a very high level of interference from the macro BTSs, unless power management is done appropriately. Since power management had been so far very simple, i.e., the power budget was divided equally among all available channels, channel allocation had to be done in a way that avoided interference (the onus was on channel allocation). For example, orthogonal deployment (OD) was proposed, that simply separates the frequency band of the small cells and the macro BTSs. Specifically, $k$ subchannels are allocated to the small cells and the remaining $M-k$ subchannels are allocated to the macro BTSs. In this case, macro BTSs do not create interference with SCs. OD was shown to work much better than a scheme where all BTSs used all the channels (reuse-1 over all BTSs) and the power budget of each BTS was shared equally over the channels [34]. In the following, we will show that OD is not a very efficient method, and that when all the BTSs operate on the same frequency band with a robust power map the system performance can be increased drastically.

User association is also more challenging in HetNets than in homogeneous networks. In homogeneous networks, a user association policy that connects a user to the BTS that yields the highest channel gain to that user is simple and very efficient. However, using such a user association in a HetNet would make most of the users to associate to the macro BTSs. An association scheme for HetNets, called Small Cell First (SCF), was proposed in [34], which we will also use in the following. For this method, only for user association purpose, we assume that all the BTSs, including the small cells, use all the subchannels with reuse- 1 and spread their power evenly over all channels and we compute the SINR seen by each user from each BTS. Then, a user associates to a small cell if the per subchannel SINR that the user receives from that small cell is greater than a threshold $\phi$. Otherwise, it associates to the cell that yields the highest SINR. SCF with reuse-1 performs very well as long as the selection of $\phi$ is done properly, as it will be shown later.

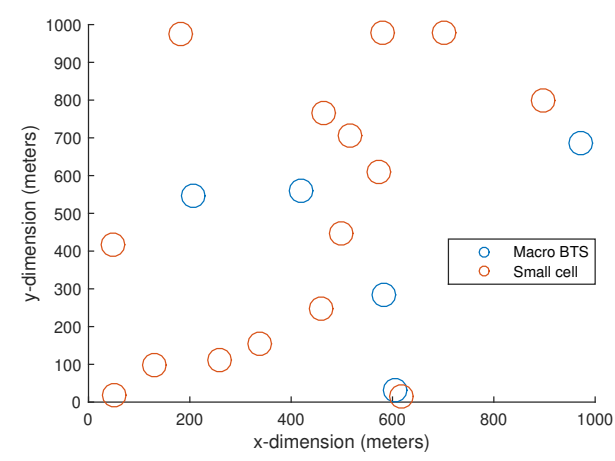

Fig. 16. The heterogeneous network topology we study with 5 macro BTSs and 15 small cells.

We consider the HetNet illustrated in Fig. 16. In our model, all macro BTSs are identical, all small cells are identical and they all belong to the same operator. We compute the power map for this HetNet with the same method described in Sec. IV-B using $|\Omega|=10$ calibration realizations with $N_{\omega}=100$ users in each calibration realization. Note that we compute a different power map for different values of the user association parameter $\phi$. Then, we compare the performance of the power map with the three benchmark schemes described in Table IV. Basically, all three schemes employ OD for channel allocation, but they allocate power differently. Default SFR is used in both the macro BTSs and the small cells in the first scheme (both schemes are parametrized independently of each other). Similar to the previous section, we determine the default SFR power allocation per type of BTSs using Voronoi cells. For the second scheme, macro BTSs use the default SFR scheme, whereas the small cells allocate equal power to all their subchannels, i.e., EP-R1. For the third scheme, both macro BTSs and small cells use EP-R1. Recall that OD is used and hence the small cells and the macro BTSs 
operate on different bands. The three schemes use the SCF user association.

TABLE IV

BENCHMARK SCHEMES FOR HETNETS

\begin{tabular}{|c|c|c|c|}
\hline & Scheme 1 & Scheme 2 & Scheme 3 \\
\hline Channel Allocation & OD & OD & OD \\
\hline Power Allocation (macro BTSs) & Default SFR & Default SFR & EP-R1 \\
\hline Power Allocation (small cells) & Default SFR & EP-R1 & EP-R1 \\
\hline
\end{tabular}

We set the power budget of the small cells to $30 \mathrm{dBm}$. The path loss between a user $u$ and a small cell $j$ is computed as $\Gamma_{u, j}=140.7+36.7 \cdot \log \left(D_{u, j} / 1000\right) \mathrm{dB}$. The antenna gain of the small cells is set to $5 \mathrm{dBi}$. Fig. 17 shows the GM throughput averaged over $|\Pi|=100$ test realizations, with $N_{\pi}=100$ users, as a function of the user association parameter $\phi$ for the three benchmark schemes as well as the power map scheme with $b=5$. For the three benchmark schemes, for each value of $\phi$, we found the value of $k$ that yielded the largest averaged GM throughput.

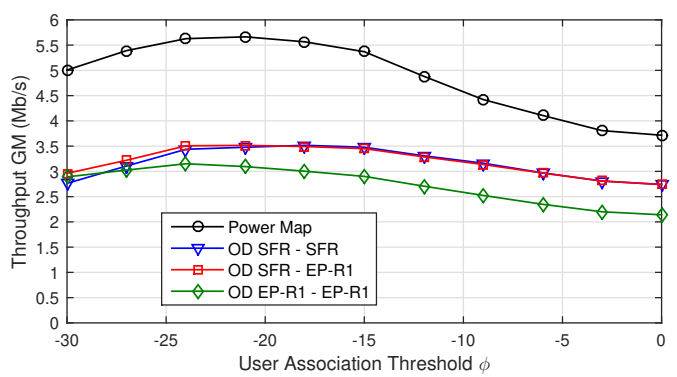

Fig. 17. GM throughput comparison for different schemes for the HetNet shown in Fig. 16, as an average of $|\pi|=100$ test realizations with $N_{\pi}=100$ users in each realization, with respect to the user association parameter $\phi$.

Clearly, the power map outperforms all the other schemes. When the best $\phi$ is selected for each scheme, the power map outperforms scheme 1, which uses default SFR for both macro BTSs and the small cells, by $58 \%$, and scheme 3 by $79 \%$. The difference in terms of the arithmetic mean throughput is $57 \%$, $58 \%$, and $69 \%$ for schemes 1,2 , and 3, respectively. Therefore, the proposed power map scheme is even more efficient for the case of HetNets.

\section{Numerical Results for the Dynamic Setting}

In the previous sections, we evaluated the power map performance on network snapshots, in which users are already in the network, and associated to the BTSs. The scheduling was done optimally. Solving these snapshot problems allowed us to verify the advantages of using a power map, as well as its robustness. In order to verify the performance of the power map in more practical scenarios, we consider in this section a dynamic network setting. Similar to the previous section, we first evaluate the performance of the power map in homogeneous networks and then in HetNets.

\section{A. Homogeneous networks}

We begin our discussion with homogeneous networks. We first propose an online scheduler and then investigate the delay performance of the proposed method. We also verify that the previous results hold for different practical user associations.

1) Dynamic settings: For a given network topology, we compute a power map with $b$ sub-bands using the planning tool described above. Hence, the power of each BTS on each sub-band is known. We assume that users appear in the network in random positions, with their arrival following a Poisson process, with an average arrival rate $\lambda\left(\mathrm{s}^{-1}\right)$. Each user must first associate to a BTS, and then download a file of fixed size $F$. The download duration for user $u$ is $t_{u}$ and, once the file is downloaded, the user leaves the network.

a) User association policies: We compare two association policies. The first policy is Best of Best SINR (given in Eq. (33)), in which a user $u$ associates to the BTS $j^{\prime}$ from which it receives the highest maximum SINR (Eq. (1)) over all the sub-bands. The second is denoted Load Aware (given in Eq. (34)), in which a user $u$ associates to the BTS $j^{*}$ in which it would see the the highest maximum ratio of the achievable per band rate (Eq. (2)) over the number of users associated to that BTS, $\left|\mathcal{U}_{j}\right|$, over all sub-bands. Unlike Best of Best SINR, this user association policy not only accounts for the peak rate a user can get from a BTS on each sub-band, but also for the BTS load, represented by the number of users associated to it.

$$
\begin{gathered}
j^{\prime}=\arg \max _{j \in \mathcal{J}, s \in \mathcal{S}}\left(\gamma_{u, j}^{s}\right) \\
j^{*}=\arg \max _{j \in \mathcal{J}, s \in \mathcal{S}}\left(\frac{R_{u, j}^{s}}{\left|\mathcal{U}_{j}\right|}\right)
\end{gathered}
$$

b) User scheduling algorithm: Once user $u$ associates to BTS $j$, the scheduling is done locally at BTS $j$, for all the users associated to it, independently of the other BTSs. In the ideal case, the users of each BTS are optimally scheduled (see Sec. V-A1b, Eq. (29)-(32)). However, in practice, the BTSs are not equipped to solve such an optimization problem fast enough. Therefore, in the following, we propose a simple opportunistic scheduling algorithm that performs quasi-optimally and can be implemented very fast. Note that Round Robin (RR) is used on all equal power schemes [34] and that, since no practical scheme has been designed for default SFR, we use our scheduling in that case as well, since default SFR is just a special case of our power map scheme.

The heuristic online scheduling we propose is performed locally by each BTS on a per-subframe basis. Recall that a frame consists of $T$ subframes. When we have the power map for all cells, we can compute the rate each user can obtain during a subframe on each sub-band. Then, instead of computing the schedule for all sub-bands and all subframes simultaneously using Eq. (29)-(32), we schedule each sub-band one by one (i.e., sequentially) at each subframe in a manner similar to RR. Let $r_{u, j}^{s}$ be the rate user $u$ of BTS $j$ receives if sub-band $s$ is allocated to that user in a subframe, and $\Psi_{u, j}$ be the total rate user $u$ of BTS $j$ has received until the allocation of this sub-band in that subframe. In the following, we assume that the whole sub-band is assigned to a user during a subframe. Then, at a given subframe $t$, if we want to decide 
to which user is allocated sub-band $s$ of BTS $j$, we need to solve the following problem:

$$
\begin{aligned}
& \max _{x_{u, j}^{s}} \sum_{u \in \mathcal{U}_{j}} \log \left(\Psi_{u, j}+x_{u, j}^{s} \cdot r_{u, j}^{s}\right) \\
& \text { s.t. } \sum_{u \in \mathcal{U}_{j}} x_{u, j}^{s}=1, \forall s \in \mathcal{S}
\end{aligned}
$$

where $x_{u, j}^{s}$ is a binary variable that determines if sub-band $s$ of BTS $j$ is allocated to user $u$ at that subframe. The objective function is the GM throughput of the users after the allocation of sub-band $s$ to a user in that subframe.

Lemma VI.1. The optimal point of the above optimization problem is the same with the optimal point of the following problem:

$$
\arg \max _{u \in \mathcal{U}_{j}} \frac{r_{u, j}^{s}}{\Psi_{u, j}}
$$

Proof of the lemma is given in Appendix B.

Using the Lemma, we design the following algorithm that schedules the users of a cell $j$ for a given subframe.

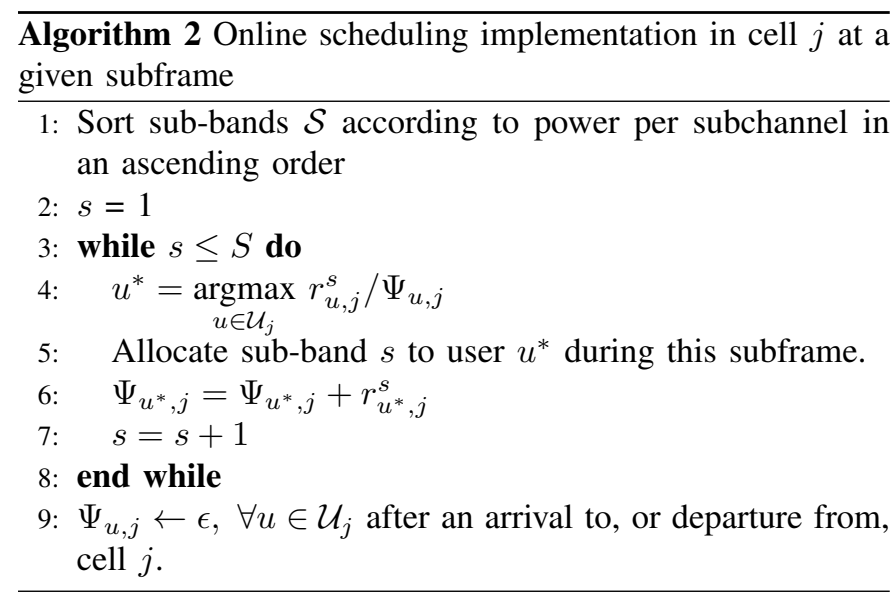

Algorithm 2 schedules each sub-band one by one using a very simple equation for a given subframe. This algorithm aims at being fair for the group of users currently in the cell, hence we set $\Psi_{u, j}$ to a very small positive number $\epsilon$ for all users in cell $j$ after an arrival or departure in cell $j$.

We illustrate the performance of the online heuristic scheduler by comparing it to the optimal scheduler (see constraints (29-32)). We consider a static setting using the homogeneous network shown in Fig. 13 and the power map with $b=3$ bands obtained for this network using $|\Omega|=$ 10 calibration realizations with $N_{\omega}=100$ users in each realization. We compare the GM throughput CDF curves of the two schedulers on $|\Pi|=200$ test realizations with $N_{\pi}=100$ users in each realization in Fig. 18.

Fig. 18 shows that the two schedulers yield almost identical GM throughput performance, and hence the online scheduling algorithm performs quasi-optimally.

2) Simulation results: We consider the topology with 5 BTS depicted in Fig. 2. We compare the average user download duration in two scenarios, 1) for the power map

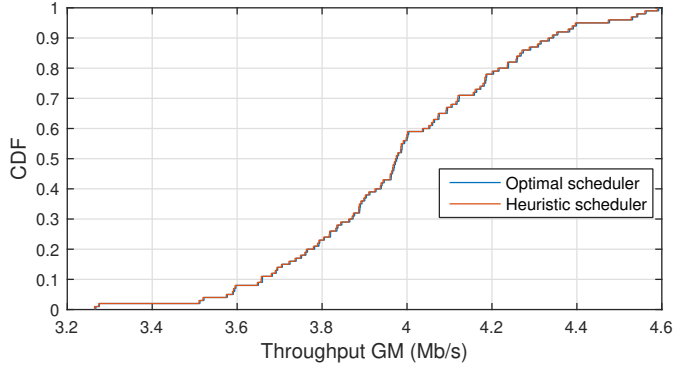

Fig. 18. CDF of the per test realization GM throughputs for the two schedulers, for the network shown in Fig. 13 over $|\pi|=200$ test realizations with $N_{\pi}=100$ users in each test realization.

with $b=5$ sub-bands (Fig. 3) and 2) the SoA benchmark (EP-R1).

Since the number of users in each cell varies over time, as the users enter and leave the network, the per-frame throughput of users changes over time. Based on the different per-frame throughputs of a user $u$, we compute the time $t_{u}$ spent by user $u$ in the network, i.e., the time it takes for $u$ to download the file of fixed size. Finally, we compute the average user delay in the network as follows:

$$
\tau=\sum_{u \in \mathcal{U}} t_{u} /|\mathcal{U}|
$$

Fig. 19 shows the average user delay $\tau$ in each of the studied cases, as a function of the average user arrival rate $\lambda$, with a confidence interval at $95 \%$. The average user delay is computed based on the batch means method, i.e., running a very long simulation, dividing it up into several batches of equal duration, then computing the mean over the batches in the steady state.

We can make at least three observations on Fig. 19. First, our power map leads to significant gains in terms of user delay, which corroborates the previous results based on snapshots. For example, the maximum arrival rate to get an average delay of $10 \mathrm{~s}$ is about 0.2 users per second with the SoA equal power/reuse 1 benchmark, while it is about 0.45 users per second for our power map (more than double). Second, as expected, the load-aware user association outperforms the Best of Best SINR association. Third, while the power map was obtained for a user association policy based on channel gains in the calibration phase, we show that this power map is performing well, even when different (more realistic) user association policies are adopted during network operation.

In summary, we propose the following complete suite of radio resource management processes for an homogeneous network: i) A planning tool to compute a power map (using Algorithm 1); ii) An online scheduling algorithm (Algorithm 2); iii) A load-aware user association given by Eq. (34). This suite of processes outperforms significantly the SoA suite based on i) EP-R1, ii) Round Robin scheduling; iii) A load-aware user association.

\section{B. Heterogeneous networks}

In the following, we will assess the performance of the power map in HetNets in the same dynamic setting as above. 


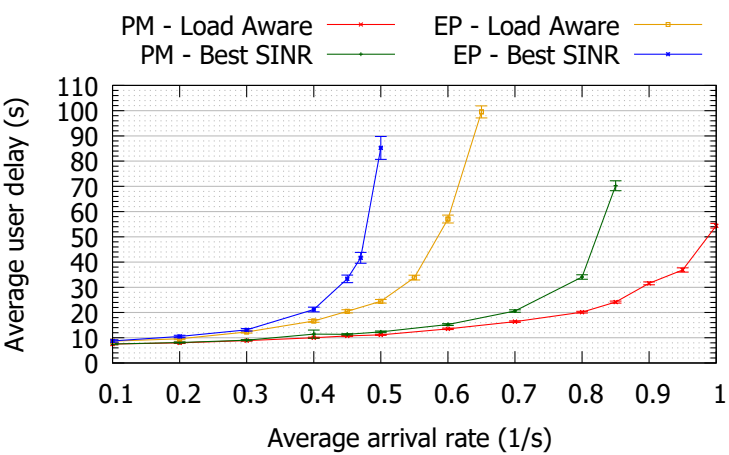

Fig. 19. Average user delay to download a file of $50 \mathrm{MB}$, as a function of the user arrival rate $\lambda$ in the network of Fig. 2, with the SoA benchmark (EP) and with the power map (PM) obtained for $|\Omega|=10$ calibration realizations, $N_{\omega}=100$, and $b=5$ sub-bands, for different user association schemes (Best of Best SINR and Load Aware).

We consider the HetNet topology in Fig. 16. We compare the performance of a benchmark OD scheme, which separates the frequency bands of the macro BTSs and the small cells, with default SFR among the macro BTSs and EP-R1 among the small cells (scheme 2 in Sec. V-B), denoted as SFR - EP-R1 below.

For user association, we use, for both schemes, a combination of SCF and Best of Best SINR. Specifically, given the parameters of the schemes (the power map or the value of $k$, the number of subchannels allocated to the small cells, for OD) as well as $\phi$ (we will discuss how $\phi$ is obtained later), we first compute the SINR values for each user from each BTS on each sub-band. Then, we associate a user to a small cell if the maximum SINR on any of the bands from that small cell is higher than the threshold $\phi$. Otherwise, we associate that user to the BTS from which it receives the highest SINR from on any of the sub-bands. Assume $\mathcal{J}_{s}$ denotes the set of small cells. Then, a user $u$ associates to cell $j^{\prime}$ according to the following rule:

$$
j^{\prime}= \begin{cases}\arg \max _{j \in \mathcal{J}_{s}, s \in \mathcal{S}}\left(\gamma_{u, j}^{s}\right), & \text { if } \max _{j \in \mathcal{J}_{s}, s \in \mathcal{S}}\left(\gamma_{u, j}^{s}\right) \geq \phi \\ \arg \max _{j \in \mathcal{J}}, s \in \mathcal{S}\left(\gamma_{u, j}^{s}\right), & \text { otherwise }\end{cases}
$$

For our method, we use the power map that we obtained in Sec. V-B with $b=5$ bands. Recall that, as shown in Fig. 17, we computed different power maps for different user association parameters. In this section, we use the one that maximizes the average GM throughput. However, since we are using a different user association policy here, we need a new value of $\phi$ for that same power map. To this end, we do a second static phase, where we now fix the power map and find the best $\phi$ for the new user association policy.

To find a robust value of $\phi$, we perform some computations again in the static setting both for the power map and the OD SFR - EP-R1 schemes. We perform computations for $|\Pi|=$ 100 test realizations with 100 users, and try different values of $\phi$ for the power map and different values of $\phi$ and $k$ for the OD SFR - EP-R1 scheme. Then, we pick the values that maximize the average GM throughput for the two schemes. For the power map, $\phi=3 \mathrm{~dB}$ gives the best results, whereas $\phi=0 \mathrm{~dB}$ and $k=48$ gives the best results for the OD SFR - EP-R1 scheme.

The average delay performance of the two schemes is given in Fig. 20 when each user arrives at the network and leaves after completing the download of a $50 \mathrm{MB}$ file. We use the same local scheduling algorithm as in Algorithm 2. ${ }^{2}$

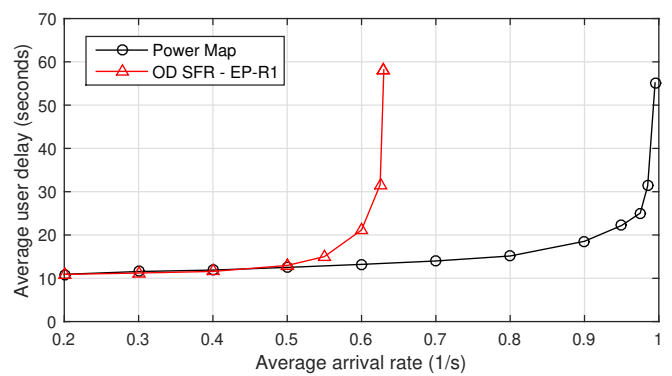

Fig. 20. Average user delay to download a file of $50 \mathrm{MB}$, as a function of the user arrival rate $\lambda$ in the network of Fig. 16, with OD SFR - EP-R1 scheme and with the power map obtained for $|\Omega|=10$ calibration realizations, $N_{\omega}=$ 100 , and $b=5$ sub-bands.

Clearly, the results of the static setting also hold for the dynamic setting, i.e., our power map scheme outperforms significantly the benchmark. For example, the power map can provide an average delay of $20 \mathrm{~s}$ to an arrival rate of 0.92 user per second, whereas this number is 0.6 users per second for the benchmark, a $50 \%$ gain.

In summary, our power map scheme performs significantly better than SoA solutions in dynamic settings as well, complemented by a fast online scheduling algorithm (Algorithm 2) and an easy to parametrize user association scheme, based on a combination of SCF and Best of Best SINR. Our results demonstrate that complex dynamic solutions are not necessarily required to outperform SFR: the power map can be computed offline, based only on network topology information, while remaining robust in dynamic scenarios.

\section{CONCLUSION}

We propose in this paper a robust tool to plan both homogeneous and heterogeneous networks with non-regular BTS placement along with the online RRM processes that go with it. More precisely, we present an offline algorithm capable of finding a robust power map, that determines the transmission power level of each BTS on the different sub-bands. This power map is computed prior to the network operation, with the sole knowledge of the network topology, and no coordination between the BTSs. The algorithm only takes as input a set of realizations, denoted as calibration realizations, representing different network snapshots. Once computed, the power map is fixed and followed by the BTSs in the network throughout its operation.

We also propose an online scheduling algorithm and an easy to use user association scheme, resulting in a full

\footnotetext{
${ }^{2}$ Note that our benchmark for the HetNets is different than the one in the homogeneous case since the benchmark we used in the homogeneous case cannot be used directly for HetNets as a solution based on a reuse 1 channel allocation among all cells (macro and small) and the same user association policy as for the homogeneous case works very poorly as shown in [34].
} 
suite of RRM processes. Numerical results show that our framework drastically outperforms both the classical reuse-1 scheme and the default SFR scheme, both in homogeneous and heterogeneous network settings.

We leave for future work, the extension to our planning techniques to other bands (e.g., mmWave) and other PHY technologies (e.g., MIMO or Non-Orthogonal Multiple Access). We believe that robust planning techniques such as the one we have proposed here are going to be even more useful in 5G, where QoS (in particular delay), reliability and availability are critical for some applications.

\section{APPENDIX A}

\section{THE COMPLEMENTARY GP PROBLEM}

Problem $\mathcal{P}_{1}$, as formulated in Sec. IV-A, is a non-convex problem with non-linear constraints. In order to solve it, we first transform it into a complementary GP, denoted $\mathcal{P}_{1}^{*}$, by re-writing its constraints to match the typical structure of a complementary GP [36], as explained in Sec. IV-B1.

$$
\begin{aligned}
& \mathcal{P}_{\mathbf{1}}^{*}: \max _{P_{j}^{s}, \lambda_{u}(\omega), \gamma_{u, j}^{s}(\omega), R_{u, j}^{s}(\omega), \alpha_{u, j}^{s}(\omega)} \mathcal{Z} \\
& \frac{\mathcal{Z} \cdot|\Omega|}{\sum_{\omega \in \Omega}\left(\prod_{u \in \mathcal{U}(\omega)} \lambda_{u}(\omega)\right)^{\frac{1}{|\mathcal{U}(\omega)|}}} \leq 1 \\
& \frac{\gamma_{u, j}^{s}(\omega) \cdot k \cdot \mathcal{N}_{0}+\gamma_{u, j}^{s}(\omega) \cdot \sum_{h \in \mathcal{J}, h \neq j} P_{h}^{s} \cdot G_{u, h}^{s}}{P_{j}^{s} \cdot G_{u, j}(\omega)} \leq 1 \\
& \forall j \in \mathcal{J}, u \in \mathcal{U}_{j}(\omega), s \in \mathcal{S}, \omega \in \Omega \\
& \frac{R_{u, j}^{s}(\omega)}{k \cdot \eta \cdot\left(\gamma_{u, j}^{s}(\omega)\right)} \leq 1, \\
& \frac{R_{u, j}^{s}(\omega)}{k \cdot R_{m a x}} \leq 1, \forall j \in \mathcal{J}, u \in \mathcal{U}_{j}(\omega), s \in \mathcal{S}, \omega \in \Omega \\
& \frac{\lambda_{u}(\omega)}{\sum_{s \in \mathcal{S}} \alpha_{u, j}^{s}(\omega) \cdot R_{u, j}^{s}(\omega)} \leq 1, \forall j \in \mathcal{J}, u \in \mathcal{U}_{j}(\omega), \omega \in \Omega \\
& \frac{\sum_{s \in \mathcal{S}} P_{j}^{s}}{P_{j}} \leq 1, \forall j \in \mathcal{J} \\
& \sum_{u \in \mathcal{U}_{j}(\omega)} \alpha_{u, j}^{s}(\omega) \leq 1, \forall s \in \mathcal{S}, j \in \mathcal{J}, \omega \in \Omega \\
& P_{j}^{s} \geq 0, \lambda_{u}(\omega) \geq 0, \gamma_{u, j}^{s}(\omega) \geq 0, R_{u, j}^{s}(\omega) \geq 0, \alpha_{u, j}^{s}(\omega) \geq 0 \\
& (44)
\end{aligned}
$$

\section{APPENDIX B}

\section{PROOF OF LEMMA VI.1}

Proof. The objective function of the optimization problem can be rewritten as follows:

$$
\max _{x_{u, j}^{s}} \sum_{u \in \mathcal{U}_{j}} \log \left(\Psi_{u, j}\right)+\sum_{u \in \mathcal{U}_{j}} \log \left(1+\frac{x_{u, j}^{s} \cdot r_{u, j}^{s}}{\Psi_{u, j}}\right)
$$

Note that the first term is a constant. Therefore, we only need to focus on maximizing the second term.

$$
\max _{x_{u, j}^{s}} \sum_{u \in \mathcal{U}_{j}} \log \left(1+\frac{x_{u, j}^{s} \cdot r_{u, j}^{s}}{\Psi_{u, j}}\right)
$$

Since only one user will receive sub-band $s$ in the subframe under consideration, $x_{u, j}^{s}$ will be zero for all other users and hence only one term of the sum will not be equal to zero. So, at the optimal point, the value of the objective function will be $\log \left(1+\frac{r_{u^{*}, j}^{s}}{T_{u^{*}, j}}\right)$, where $u^{*}$ is the user sub-band $s$ is allocated to. Therefore, the objective function is maximized when the user that maximizes the $\log \left(1+\frac{r_{u, j}^{s}}{\Psi_{u, j}}\right)$ term receives sub-band $s$. This is equivalent to finding the user with maximum $\frac{r_{u, j}^{s}}{\Psi_{u, j}}$ among all users in $\mathcal{U}_{j}$, which is the same as Eq. (37).

\section{REFERENCES}

[1] 3GPP TSG RAN WG1 Meeting \#41, "Soft Frequency Reuse Scheme for UTRAN LTE", R1-050507, May 2005.

[2] 3GPP TSG RAN WG1 Meeting \#42, "Further Analysis of Soft Frequency Reuse Scheme", R1-050841, Sep. 2005.

[3] F. Baccelli, M. Klein, M. Lebourges, S. Zuyev, "Stochastic Geometry and Architecture of Communication Networks", Telecommunication Systems, 7(1), June 1997.

[4] J. G. Andrews et al, "A Tractable Approach to Coverage and Rate in Cellular Networks”, IEEE Trans. on Communications, 59(11), 2011.

[5] L. Chen, D. Yuan, "Soft Frequency Reuse in Large Networks with Irregular Cell Pattern: How Much Gain to Expect?", IEEE PIMRC, 2009.

[6] R. Kwan, C. Leung, "A Survey of Scheduling and Interference Mitigation in LTE”, Journal of Electrical and Comp. Engineering, 2010.

[7] A. Hamza, S. Khalifa, H., Hamza, K. Elsayed, "A Survey on Inter-cell Interference Coordination Techniques in OFDMA-based Cellular Networks", IEEE Comm. Surveys \& Tutorials, 15(4), Mar. 2013.

[8] Y. Hu, M. Ozmen, M. C. Gursoy and A. Schmeink, "Optimal Power Allocation for QoS-Constrained Downlink Multi-User Networks in the Finite Blocklength Regime," IEEE Transactions on Wireless Communications, vol. 17, no. 9, pp. 5827-5840, Sept. 2018.

[9] Y. Ozcan and C. Rosenberg, "Revisiting Downlink Scheduling in a Multi-Cell OFDMA Network: From Full Base Station Coordination to Practical Schemes", Wireless Days Conference, April 2019.

[10] I. G. Fraimis, V. D. Papoutsis, S. A. Kotsopoulos, "A Decentralized Subchannel Allocation Scheme with Inter-Cell Interference Coordination (ICIC) for Multi-Cell OFDMA Systems," IEEE GLOBECOM, 2010.

[11] A. Triki, L. Nuaymi, "Inter-cell Interference Coordination Algorithms in OFDMA Wireless Systems", IEEE Vehicular Technology Conference (VTC), May 2011.

[12] L. Venturino, A. Zappone, C. Risi and S. Buzzi, "Energy-Efficient Scheduling and Power Allocation in Downlink OFDMA Networks With Base Station Coordination," IEEE Transactions on Wireless Communications, vol. 14, no. 1, pp. 1-14, Jan. 2015.

[13] T. Zhou, Z. Liu, J. Zhao, C. Li and L. Yang, "Joint User Association and Power Control for Load Balancing in Downlink Heterogeneous Cellular Networks," IEEE Transactions on Vehicular Technology, vol. 67, no. 3, pp. 2582-2593, March 2018.

[14] W. Zhang, Y. Wang and P. Li, "Coordinated resource allocation with fractional frequency reuse for downlink OFDMA networks," IEEE Wireless Communications and Networking Conference (WCNC), 2014.

[15] G. Li and H. Liu, "Downlink Radio Resource Allocation for Multi-Cell OFDMA System," IEEE Transactions on Wireless Communications, vol. 5, no. 12, pp. 3451-3459, December 2006.

[16] Mohammed Yazid Lyazidi, Nadjib Aitsaadi, Rami Langar, "A dynamic resource allocation framework in LTE downlink for Cloud-Radio Access Network", Computer Networks, Volume 140, 2018.

[17] Y. Wang and Q. Zhu, "Modeling and Analysis of Small Cells Based on Clustered Stochastic Geometry," IEEE Communications Letters, vol. 21, no. 3, pp. 576-579, March 2017.

[18] I. Atzeni, J. Arnau and M. Kountouris, "Downlink Cellular Network Analysis With LOS/NLOS Propagation and Elevated Base Stations," IEEE Transactions on Wireless Communications, vol. 17, no. 1, pp. 142-156, Jan. 2018.

[19] H. ElSawy, A. Sultan-Salem, M. Alouini and M. Z. Win, "Modeling and Analysis of Cellular Networks Using Stochastic Geometry: A Tutorial," IEEE Communications Surveys \& Tutorials, vol. 19, no. 1, pp. 167-203, Firstquarter 2017.

[20] G. Boudreau, J. Panicker, N. Guo, R. Chang, N. Wang, S. Vrzic, "Interference Coordination and Cancellation for 4G Networks", IEEE Communications Magazine, 47(4), Apr. 2009. 
[21] K. Begain, G. I. Rozsa, A. Pfening, M. Telek, "Performance Analysis of GSM Networks with Intelligent Underlay Overlay", IEEE Symposium on Computers and Communications (ISCC), 2002.

[22] M. Sternad, T. Ottosson, A. Ahlen and A. Svensson, "Attaining Both Coverage and High Spectral Efficiency with Adaptive OFDM Downlinks", IEEE Vehicular Technology Conference (VTC), Oct. 2003.

[23] J. Li, N. B. Shroff and E. K. P. Chong, "A Reduced-power Channel Reuse Scheme for Wireless Packet Cellular Networks", IEEE/ACM Trans. on Networking, 1999.

[24] Masood Maqbool, Philippe Godlewski, Marceau Coupechoux, Jean-Marc Kélif, "Analytical Performance Evaluation of Various Frequency Reuse and Scheduling Schemes in Cellular OFDMA Networks", Performance Evaluation, 67(4), 2010.

[25] X. Mao, A. Maaref, K. H. Teo, " Adaptive Soft Frequency Reuse for Inter-cell Interference Coordination in SC-FDMA Based 3GPP LTE Uplinks", IEEE GLOBECOM, Nov. 2008.

[26] A. Damnjanovic et al., "A survey on 3GPP heterogeneous networks," IEEE Wireless Communications, vol. 18, no. 3, pp. 10-21, June 2011.

[27] H. Tran, G. Kaddoum, H. Tran and E. Hong, "Downlink Power Optimization for Heterogeneous Networks With Time Reversal-Based Transmission Under Backhaul Limitation,” IEEE Access, vol. 5, pp. 755-770, 2017.

[28] K. Shen, Y. Liu, D. Y. Ding and W. Yu, "Flexible Multiple Base Station Association and Activation for Downlink Heterogeneous Networks," IEEE Signal Processing Letters, vol. 24, no. 10, Oct. 2017.

[29] S. Jabeen and P. Ho, "Joint User Scheduling and RRU Association for ODFMA-Based Networks With Inter-BBU Coordination," IEEE Trans. on Communications, vol. 67, no. 5, pp. 3638-3650, May 2019

[30] A. Abdelnasser, E. Hossain and D. I. Kim, "Tier-Aware Resource Allocation in OFDMA Macrocell-Small Cell Networks," IEEE Trans. on Communications, vol. 63, no. 3, pp. 695-710, March 2015.

[31] J. Miao et al., "Joint Power and Bandwidth Allocation Algorithm with QoS Support in Heterogeneous Wireless Networks," IEEE Communications Letters, vol. 16, no. 4, pp. 479-481, April 2012.

[32] A. R. Elsherif, W. Chen, A. Ito and Z. Ding, "Adaptive Resource Allocation for Interference Management in Small Cell Networks," IEEE Trans. on Communications, vol. 63, no. 6, pp. 2107-2125, June 2015.

[33] H. Zhang, H. Liu, J. Cheng and V. C. M. Leung, "Downlink Energy Efficiency of Power Allocation and Wireless Backhaul Bandwidth Allocation in Heterogeneous Small Cell Networks," IEEE Transactions on Communications, vol. 66, no. 4, pp. 1705-1716, April 2018.

[34] D. Fooladivanda, C. Rosenberg," "Joint Resource Allocation and User Association for Heterogeneous Wireless Cellular Networks", IEEE Transactions on Wireless Communications, 12(1), Jan. 2013.

[35] S. Boyd, S.J. Kim, L. Vandenberghe, A. Hassibi, "A Tutorial on Geometric Programming", Optimization and Engineering, 8(1), 2007.

[36] M. Chiang, C. Tan, D. Palomar, O. Daniel, D. Julian, "Power Control by Geometric Programming", IEEE Transactions on Wireless Communications, 6(7), July 2007.

[37] 3GPP TSG RAN WG1, "Evolved Universal Terrestrial Radio Access (E-UTRA); Further Advancements for E-UTRA Physical Layer Aspects (Release 9)", TR 36.814, Mar. 2010.

[38] Bonmin: Basic Open-source Nonlinear Mixed Integer Programming, Available at http://www.coin-or.org/Bonmin

[39] K. Doppler, C. Wijting, K. Valkealahti, "Interference Aware Scheduling for Soft Frequency Reuse", IEEE Vehicular Technology Conference (VTC), Apr. 2009

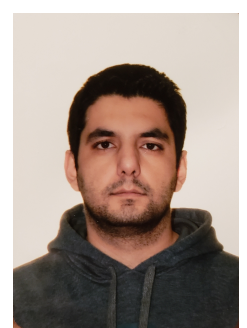

Yigit Özcan received his B.Sc. and M.Sc. degrees in Electrical and Electronics Eng. dept. of Middle East Technical University, Turkey in 2010 and 2013, respectively and he received his Ph.D. degree in Electrical and Computer Eng. dept. of University of Waterloo, Canada in 2019. He is now working as a Wireless System Engineer in Blinq Networks, Canada. His research interests are resource allocation in wireless networks and networking protocols.

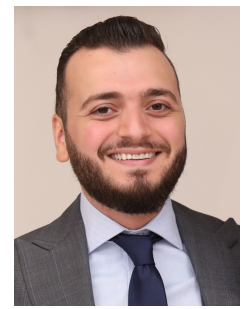

Jad Oueis received his Ph.D and M.Sc. degrees in Computer Science and Networking from INSA Lyon (France), in 2018 and 2015, respectively. He also holds a M.Eng. degree in Telecommunication from the Lebanese University. $\mathrm{He}$ is currently working as a digital innovation consultant, and his research interests are focused on mobile and wireless networks.

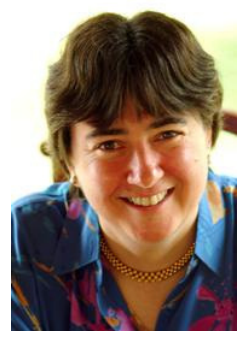

Catherine Rosenberg, FIEEE is a Professor with the Department of Electrical and Computer Engineering at the University of Waterloo and the Canada Research Chair in the Future Internet. She is a Fellow of the Canadian Academy of Engineering. Her research interests are in networking, wireless, and energy systems. More information is available at https://ece.uwaterloo.ca/ cath/.

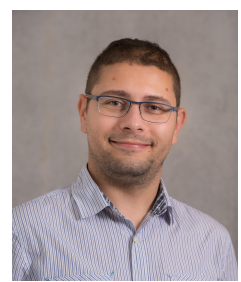

Razvan Stanica is associate professor at INSA Lyon, France, and a research scientist with the INRIA Agora team of the CITI laboratory. He obtained a M.Eng. degree and a Ph.D. in computer science, both from INP Toulouse, France, in 2008 and 2011 respectively. His research interests include wireless mobile networks, with a special focus on communication networks in urban environments.

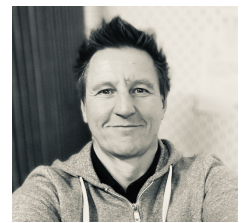

Fabrice Valois is full professor at INSA Lyon (France), since 2008. In January 2000, he received a $\mathrm{Ph} . \mathrm{D}$. in computer science from University of Versailles (France). In November 2007, he received the Habilitation á Diriger des Recherches from University of Lyon I and INSA Lyon. His research interests are in the area of networking protocols and architectures for cellular and wireless networks. 